\title{
Flow transitions and combined free and forced convective heat transfer in rotating curved channels: The case of positive rotation
}

\author{
Liqiu Wanga) \\ School of Mechanical \& Production Engineering, Nanyang Technological University, 639798 Singapore \\ K. C. Cheng \\ Department of Mechanical Engineering, University of Alberta, Edmonton, Alberta T6G 2G8, Canada
}

(Received 11 July 1995; accepted 29 January 1996)

\begin{abstract}
The simultaneous effects of curvature, rotation and heating/cooling in channel flow complicate the flow and heat transfer characteristics beyond those observed in the channels with simple curvature or rotation. The phenomena encountered are examined for steady, hydrodynamically and thermally fully developed flow in square channels. The governing equations are solved numerically by using a finite-volume method. Certain hitherto unknown flow patterns are found. And the results show both the nature of the flow transition and the effect of this transition on the distributions of temperature, friction factor and Nusselt number in a square channel. (C) 1996 American Institute of Physics. [S1070-6631(96)00406-X]
\end{abstract}

\section{INTRODUCTION}

Fluid flow and heat transfer in rotating curved channels are encountered in cooling systems for conductors of electric generators and generator motors for pumped-storage stations. They are also employed in applications such as separation processes, heat exchangers and physiological field. The transport and flow phenomena in the rotating curved channels have, therefore, challenged engineers and scientists for some time. A remarkable characteristic of the flow and heat transfer in a rotating system is the presence of the centrifugal and Coriolis forces. Under certain conditions, these forces may induce a secondary flow in a plane perpendicular to the direction of the main flow. This could significantly affect the resistance to the fluid flow and convective heat transfer.

According to its inducing condition, ${ }^{1}$ the secondary flow could be created by the Coriolis force for a constant property fluid, while the centrifugal force is purely hydrostatic, analogous to the Earth's gravitational field. When a temperatureinduced variation of fluid density occurs, both Coriolis and centrifugal-type buoyancy force could contribute to the generation of the secondary flow. On the other hand, secondary flow also arises due to centrifugal force when a channel is curved. ${ }^{2}$ Therefore, centrifugal, Coriolis and centrifugal-type buoyancy forces all contribute to the generation of the secondary flow if the channel is curved, rotated and heated/ cooled. The nonlinear interaction of these body forces with the other forces in the flow field may result in a complicated structure of the secondary flow. We attempt to examine this structure and its effects on pressure-driven main flow and temperature field in a square channel by using a finitevolume method.

The secondary flow under consideration is essentially a nonlinear combination of the buoyancy force-driven secondary flow in the mixed-convection problem, the centrifugal force-driven secondary flow in the Dean problem and the Coriolis force-driven secondary flow in straight channels

\footnotetext{
a)Telephone: (65)799-5563; fax: (65)791-1859; electronic mail: mlwang@ntuvax.ntu.ac.sg
}

with spanwise rotation (hereinafter referred to as the Coriolis problem). The similarity among these three problems has been recognized by a number of investigators. The dynamical parameters for these three problems are $D_{m}$ ( $D_{m}=R e R a$, the product of the Reynolds number Re and Rayleigh number $R a$ ), square of the Dean number $D e^{2}$ ( $D e=R e \sqrt{\sigma}$, a combination of the Reynolds number $R e$ and the curvature ratio of the channel $\sigma$ ) and $D_{\Omega}$ ( $D_{\Omega}=R e R e_{\Omega}$, the product of the Reynolds number $R e$ and rotational Reynolds number $\left.R e_{\Omega}\right)$, respectively. ${ }^{3,4}$ Here $R e=W_{m} d_{h} / \nu\left(W_{m}\right.$ is the axial mean velocity, $d_{h}$ is the hydrodynamic diameter of the channel and $\nu$ is the kinematic viscosity), $R a=\beta g \triangle T d_{h}^{3} /(\nu \alpha)$ ( $\beta$ is the thermal-expansion coefficient, $g$ is the gravitational acceleration, $\triangle T$ is the characteristic temperature difference and $\alpha$ is the thermal diffusivity), $\sigma=d_{h} / R_{c}$ ( $R_{c}$ is the curvature radius, Fig. 1) and $R e_{\Omega}=\Omega d_{h}^{2} / \nu$ ( $\Omega$ is the angular speed of rotation). Depending on the value of the dynamical parameter, fullydeveloped secondary flow exhibits three different structures for all three problems in the laminar flow region as follows.

At a relatively small value of the dynamical parameter, it consists of one-pair of counter-rotating vortices in a plane perpendicular to the axis of the channel. Upon increasing the value of the dynamical parameters sufficiently (depending on the value of the Prandtl number $\operatorname{Pr}$, curvature ratio $\sigma$ or the rotational Reynolds number $R e_{\Omega}$, respectively, for these three problems), the centrifugal, Coriolis or buoyancy force instability may lead this one-pair vortex flow structure to another form of two-dimensional flow with a two-pair or roll-cell vortex structure (depending on the geometry of the channel) in the cross-plane. Such two-pair vortex flows are found to be unstable to asymmetric perturbations in the Dean problem, ${ }^{5}$ in the Coriolis problem ${ }^{6}$ and in the mixedconvection problem. ${ }^{7}$ Upon increasing the dynamical parameter further, all two-dimensional flows become unstable, and there are evidences for the evolution of streamwise periodic three-dimensional flows in all of the three problems. ${ }^{6-8}$ The reference concerning these three problems may be found, for example, in Nandakumar and Weinitschke ${ }^{7}$ for the mixed- 


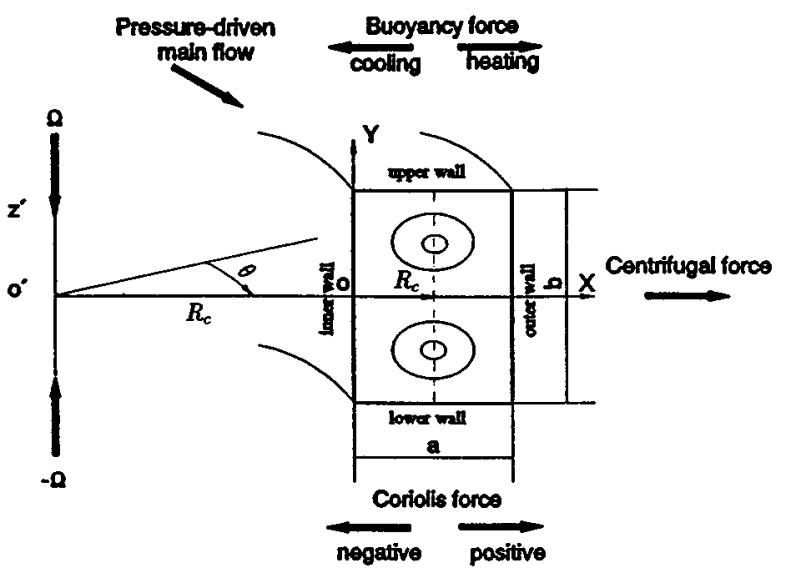

FIG. 1. Physical problem and rotating toroidal coordinate system.

convection problem; Nandakumar and Masliyah ${ }^{9}$ and Hwang and $\mathrm{Chao}^{10}$ for the Dean problem; Nandakumar et al., ${ }^{6}$ and Yang et al. ${ }^{11}$ for the Coriolis problem.

A combination of two or all of these three problems arises in many engineering fields. This has stimulated the interest on the flow and heat transfer under the combined effect of two or all of three body forces. Because of the complexities of the problem, early works have been constrained to some simplified limiting cases in which there exists one dominated body force. Under the condition that flow is fully developed and laminar, combined Dean and Coriolis problem was theoretically examined by Hocking ${ }^{12}$ and Ludwieg $^{13}$ for a rectangular or square channel with strong spanwise rotation, and Ito and Motai ${ }^{14}$ for a circular curved tube with weak spanwise rotation. Miyazaki ${ }^{15,16}$ analyzed the fully developed laminar flow and heat transfer in a curved circular/rectangular channel with spanwise rotation and heating effect by a finite-difference method. Because of the convergence difficulties of the iterative method used, no solutions in the range where three forces are of comparable order of magnitude could be obtained. Besides, the examination of the governing equations employed by Miyazaki shows that some errors existed in the viscous terms.

Since the solution is only for the asymptotic cases of slow and rapid rotation, the secondary flow revealed by the works mentioned above consists of one-pair of counterrotating vortices in a plane perpendicular to the axis of the channel. The interaction of the secondary flow with the pressure-driven main flow shifts the location of the maximum axial velocity away from the center of the channel and in the direction of the secondary velocities in the middle of the channel. When the three forces are of comparable order of magnitude, a complicated structure of the secondary flow might be expected since then the nonlinear effects could be quite strong.

More comprehensive studies have been made by Thangam and Speziale ${ }^{17}$ and Matsson and Alfredsson. ${ }^{18}$ Thangam and Speziale ${ }^{17}$ numerically examined the effect of sidewall heating in the pressure-driven laminar flow of an incompressible viscous fluid through a straight rectangular channel under a spanwise rotation. The secondary flow was found to be unicellular or multicellular, depending on the rate of heating and rotation. The work also clearly shows the heat transfer enhancement due to the rotation for a straight rectangular channel with a high aspect ratio for a range of spanwise rotation rates. Matsson and Alfredsson ${ }^{18}$ visualized and measured the flow in a curved air channel with a high aspect ratio and a spanwise rotation using smoke visualization and hot-wire measurements with an emphasis on the effect of spanwise rotation on the primary and secondary instabilities in curved channel flow. When the Coriolis force enhanced the centrifugal force (positive rotation), the vortex pairs were observed to split and merge. When the Coriolis force counteracted the centrifugal force (negative rotation), the primary Dean instability in forms of Dean vortices could be cancelled. As well, a high negative rotation rate could lead the vortices to appear on the inner convex channel wall.

In this work, we present a relatively comprehensive numerical study on the laminar flow and combined free and forced convective heat transfer in a rotating curved square channel at low to relatively rapid rotation rates where both the convective and diffusive terms play an important role and, consequently, the full nonlinear equations must be solved. Attention is focused on the flow transitions of secondary flow and main flow in the fully developed region and the effects of the flow transitions on temperature distribution, friction factors and Nusselt number for a wide range of characteristic parameters. The emphasis is also placed on the primary instabilities (centrifugal, Coriolis and buoyancy force instabilities) arising in the rotating curved channels, and not their secondary instabilities or the transition to turbulence, although the vortices studied ultimately have an important influence on the transition. The motivation for the present study arose from the following observations: (1) There exists no detailed study of the flow and heat transfer in a channel with simultaneous effects of curvature, rotation and heating/cooling. While flow and heat transfer under three effects resemble those with only two or one factors when one or two body forces are relatively weak (this is the case considered by Miyazaki ${ }^{15,16}$ ), they are endowed with some more complex features due to the nonlinearity of the problem and the nonuniform interactions of these forces over the flow domain, especially when three forces are of comparable order of magnitude; and (2) the transitions in flow structure due to the action of centrifugal, Coriolis and buoyancy forces have not been fully studied. In particular, the disappearance and reappearance of Dean-vortices, Coriolis-vortices and buoyancy-vortices have in general received less attention in the past.

\section{FORMULATION OF THE PROBLEM}

The geometrical configuration of the physical model for a rotating curved rectangular channel and its coordinate system are illustrated in Fig. 1. A viscous fluid is allowed to flow through a channel of rectangular cross section with width $\times$ height $=a \times b$ under the action of the pressure gradient along the channel axis (streamwise direction). The channel is uniformly curved around the axis $o^{\prime} z^{\prime}$, and rotates about the axis with a constant angular velocity $\Omega$. The rotation can be positive or negative as shown in Fig. 1 in terms of angular velocity vector. A positive rotation gives 
rise to a Coriolis force in the cross-plane that is directed along the positive $X$-direction, and vice versa. In addition to the curvature and rotation, the channel is being uniformly heated or cooled at the wall with a uniform peripheral temperature. The properties of the fluid, with the exception of density, are taken to be constant. To facilitate the discussion, each side of the channel wall is termed the inner, outer, upper and lower walls as shown in the figure.

Consider a non-inertial toroidal coordinate system $(O, X, Y, \theta)$ fixed to the curved channel rotating with a constant angular velocity $\Omega$ about $o^{\prime} z^{\prime}$ axis, as shown in Fig. 1. The radial (normal), spanwise and streamwise (axial) directions are $(X, Y, \theta)$, respectively. The direction of the relative velocity of the fluid in the channel is chosen in the direction of increasing $\theta$, while the angular velocity of the channel is taken as $\Omega>0$ for increasing $\theta$ (positive rotation) and $\Omega<0$ for decreasing $\theta$ (negative rotation), respectively. In order to facilitate the numerical programming, the origin of the coordinate system is located at the center of the inner wall instead of the center of the cross section.

The flow is assumed to be laminar and steady. Let $U, V$ and $W$ be the velocity components in the directions of $X, Y$ and $\theta$, respectively, and $T, T_{w}$ be the temperature of the fluid and the wall. By using the usual Boussinesq approximation to deal with the density variation, the continuity, Navier-Stokes and energy equations governing the fullydeveloped laminar flow and heat transfer are given in terms of the dimensionless variables as ${ }^{3}$ the following. continuity equation:

$$
\begin{aligned}
& \frac{\partial}{\partial x}\left(\left(1+\sigma \frac{4 x-(1+1 / \gamma)}{4}\right) u\right) \\
& +\frac{\partial}{\partial y}\left(\left(1+\sigma \frac{4 x-(1+1 / \gamma)}{4}\right) v\right)=0 ;
\end{aligned}
$$

momentum equations:

$$
\begin{gathered}
u \frac{\partial u}{\partial x}+v \frac{\partial u}{\partial y}-\frac{16 D k^{2} w^{2}}{\sigma(1+\sigma(x-(1+1 / \gamma) / 4))}-\frac{4 L_{1} D k^{2} w}{3 \sigma} \\
-16 D k L_{2}\left(1+\sigma \frac{4 x-(1+1 / \gamma)}{4}\right) t \\
=-\frac{\partial p}{\partial x}+\left(\frac{\partial^{2} u}{\partial x^{2}}+\frac{\partial^{2} u}{\partial y^{2}}+\frac{\sigma}{1+\sigma(x-(1+1 / \gamma) / 4)} \frac{\partial u}{\partial x}\right. \\
\left.\quad-\frac{\sigma^{2} u}{(1+\sigma(x-(1+1 / \gamma) / 4))^{2}}\right), \\
u \frac{\partial v}{\partial x}+v \frac{\partial v}{\partial y}=-\frac{\partial p}{\partial y}+\left(\frac{\partial^{2} v}{\partial x^{2}}+\frac{\partial^{2} v}{\partial y^{2}}\right. \\
\left.\quad+\frac{\sigma}{1+\sigma(x-(1+1 / \gamma) / 4)} \frac{\partial v}{\partial x}\right), \\
u \frac{\partial w}{\partial x}+v \frac{\partial w}{\partial y}+\frac{\sigma u w}{1+\sigma(x-(1+1 / \gamma) / 4)}+\frac{\sigma L_{1} u}{12} \\
=\frac{1}{1+\sigma(x-(1+1 / \gamma) / 4)}+\left(\frac{\partial^{2} w}{\partial x^{2}}+\frac{\partial^{2} w}{\partial y^{2}}\right.
\end{gathered}
$$

$$
\begin{aligned}
& +\frac{\sigma}{1+\sigma(x-(1+1 / \gamma) / 4)} \frac{\partial w}{\partial x} \\
& \left.-\frac{\sigma^{2} w}{(1+\sigma(x-(1+1 / \gamma) / 4))^{2}}\right)
\end{aligned}
$$

energy equation:

$$
\begin{aligned}
u \frac{\partial t}{\partial x} & +v \frac{\partial t}{\partial y}-\frac{4 D k w}{\sigma P r(1+\sigma(x-(1+1 / \gamma) / 4))} \\
& =\frac{1}{\operatorname{Pr}}\left(\frac{\partial^{2} t}{\partial x^{2}}+\frac{\partial^{2} t}{\partial y^{2}}+\frac{\sigma}{1+\sigma(x-(1+1 / \gamma) / 4)} \frac{\partial t}{\partial x}\right) .
\end{aligned}
$$

The dimensionless variables are defined as

$$
\begin{aligned}
& x=\frac{X}{d_{h}} ; \quad y=\frac{Y}{d_{h}} ; \\
& u=\frac{d_{h} U}{\nu} ; \quad v=\frac{d_{h} V}{\nu} ; \quad w=\frac{W}{W_{1}} ; \\
& p=\frac{P}{\rho\left(\nu / d_{h}\right)^{2}} ; \quad t=\frac{T_{w}-T}{\triangle T},
\end{aligned}
$$

where $\nu$ and $\rho$ are the kinematic viscosity and the density of the fluid, $d_{h}$ is the hydrodynamic diameter defined as $d_{h}=2 a b /(a+b), P$ is a pseudo pressure which absorbs any force residual implied by using the Boussinesq approximation $\left(P=p^{\prime}-\rho\left[X-a / 2+(X-a)^{2} /\left(2 R_{c}\right)\right] R_{c} \Omega^{2}\right.$ with $p^{\prime}$ as the fluid pressure and $R_{c}$ as the curvature radius), ${ }^{1,3,16}$ and $W_{1}$ and $\triangle T$ are the representative axial velocity and temperature difference, respectively, which are defined as

$$
W_{1}=\frac{d_{h}^{2} c_{1}}{\mu} ; \quad \triangle T=\operatorname{Prd}_{h} c_{2} .
$$

Here $\mu$ is the viscosity of the fluid, $P r$ is the Prandtl number, $c_{1}$ is the axial pressure gradient which is a positive constant for hydrodynamically fully developed flow $\left(c_{1}=-\partial p^{\prime} / R_{c} \partial \theta\right),{ }^{3,16}$ and $c_{2}$ is the axial temperature gradient which is a constant for the thermally fully developed flow, but can be positive and negative depending on heating or cooling of the fluid $\left(c_{2}=\partial T / R_{c} \partial \theta\right) .{ }^{3,19,20}$

It is customary to use the mean axial velocity $W_{m}$ and the difference between the wall temperature and the bulk mean temperature $\left(T_{w}-T_{b}\right)$ for the non-dimensionalization of the axial velocity and temperature, respectively. However, the employment of these quantities results unavoidably in the appearance of two unknown dimensional parameters in the governing equations which comprise the unknowns $W_{m}$ and $T_{b}$, respectively. Consequently, the iterative procedure should be applied, assuming some initial estimated values to them. It requires an additional computation time. In order to avoid this additional increase in computation time, we follow Miyazaki ${ }^{15,16}$ in using $W_{1}$ and $\triangle T$ as the representative axial velocity and the representative temperature difference, respectively. They involve the axial pressure gradient $c_{1}$ and the axial temperature gradient $c_{2}$, which are usually given as design parameters so that it does not induce a difficulty in using the computation results for design. The velocity $W_{1}$ is proportional to the pressure drop in the axial direction. For 
the flow in a stationary straight circular tube, the mean axial velocity $W_{m}$ is related to $W_{1}$ as $W_{m}=W_{1} / 8$ (Miyazaki ${ }^{15,16}$ ). The temperature difference $\Delta T$ is, on the other hand, proportional to the fluid temperature difference between the channel inlet and outlet.

Six dimensionless parameters are defined as

$$
\begin{aligned}
& \gamma=\frac{a}{b} ; \quad \sigma=\frac{d_{h}}{R_{c}} ; \quad \operatorname{Pr}=\frac{\nu}{\alpha} ; \\
& D k=\frac{\sigma d_{h} W_{1}}{4 \nu} ; \quad L_{1}=\frac{3 R e_{\Omega}}{2 D k} ; \quad L_{2}=\frac{R a_{\Omega}}{16 D k},
\end{aligned}
$$

in which

$$
R e_{\Omega}=\frac{d_{h}^{2} \Omega}{\nu} ; \quad R a_{\Omega}=\frac{\beta R_{c} \Omega^{2} d_{h}^{3} \triangle T}{\nu^{2}},
$$

with $\beta$ as the thermal-expansion coefficient.

The dimensionless groups adopted here are those in Miyazaki ${ }^{15,16}$ and Morris. ${ }^{1}$ The $\gamma$ is a geometry parameter denoting the aspect ratio of a rectangular cross section. The curvature ratio $\sigma$ is also a geometry parameter, representing the degree of curvature. The Prandtl number Pr, a thermophysical property parameter, represents the ratio of momentum diffusion rate to that of thermal diffusion. $D k$ is a pseudo Dean number with $W_{1}$ as the characteristic velocity. ${ }^{15,16}$ The rotational Reynolds number $R e_{\Omega}$ emerges from the Coriolis term of the momentum equations. It indicates ratio of the Coriolis force to the viscous force. A positive $R e_{\Omega}$ represents the case of positive rotation. A negative $R e_{\Omega}$ is for the case of negative rotation. It is noted that in the literature the rotational Reynolds number is also known as the Taylor number or the reciprocal of Ekman number. It is adopted here instead of the Ekman number because the increase in $R e_{\Omega}$ implies the growth of the Coriolis force, and its effects are more readily conceivable (in particular for the limiting case as $\Omega \rightarrow 0$ ). The rotational Rayleigh number $R a_{\Omega}$ has its origin in the centrifugal buoyancy terms. It is similar to the Rayleigh number $R a$ encountered in the study of gravitational buoyancy due to the Earth's gravitational field but with the gravitational acceleration replaced by the centrifugal acceleration measured at the center line of the channel. It denotes the ratio of the centrifugal-type buoyancy force to the viscous force. A positive $R a_{\Omega}$ represents the heating case while a negative $R a_{\Omega}$ is for the case of cooling. It is noted that the Rayleigh number is being used more and more instead of the Grashof number. ${ }^{21}$

Different scaling quantities in the nondimensionalization may result in different parameter groups for the effects of rotation, curvature and heat/cooling. Here we introduce the $L_{1}$ and $L_{2}$ to represent the effects of rotation and heating/cooling, respectively, because they represent the ratios of the dynamical parameters $D_{\Omega}$ (in the Coriolis problem) and $D_{m}$ (in the mixed-convection problem) to the parameter $D e^{2}$ in the Dean problem. ${ }^{22}$ They were found to be two parameters in determining the flow patterns in the channel with curvature, rotation and heating/cooling, ${ }^{3,14,23}$ and are introduced in the governing equations (1)-(5) explicitly since we are mainly concerned with the transition of the flow structures in this work. Note that they are related to the
Dean number, the rotational Reynolds number and the rotational Rayleigh number in an explicit way, there should be no difficulty in application of results. In particular, $R o=2 D k L_{1} /(3 R e)$ and $R a_{\Omega}=16 D k L_{2}$. Here $R o$ is the rotation number defined as $R o=\Omega d_{h} / W_{m}$.

In this work, we attempt to examine the transition of symmetric secondary flow with respect to the horizontal centerline only. Thus it suffices to consider the upper half region alone for the analysis. Then the boundary conditions may be written, in terms of dimensionless variables, as

$$
\begin{gathered}
u=v=w=t=0, \text { at } x=0, \quad \frac{1}{2}\left(1+\frac{1}{\gamma}\right), \\
\text { for } 0 \leqslant y \leqslant \frac{1}{4}(1+\gamma), \\
u=v=w=t=0, \quad \text { at } y=\frac{1}{4}(1+\gamma), \\
\text { for } 0 \leqslant x \leqslant \frac{1}{2}\left(1+\frac{1}{\gamma}\right), \\
\frac{\partial u}{\partial y}=\frac{\partial w}{\partial y}=\frac{\partial t}{\partial y}=0, \quad v=0, \quad \text { at } y=0, \\
\text { for } 0 \leqslant x \leqslant \frac{1}{2}\left(1+\frac{1}{\gamma}\right) .
\end{gathered}
$$

Within the scope of the present study, the equations (1)(5) under the boundary conditions (6)-(8) constitute the mathematical model of the problem under consideration. The imposition of the symmetry condition about the horizontal centerline constrains us to consider the symmetric solution only. However, the instabilities of the physical problem (at the various orders, secondary and the higher order) would suggest the existence of the asymmetric solutions in some region of the parameter space, the region with the high dynamical parameters in particular. The flow occurring in an experiment may, therefore, be non-symmetric in those regions. As found numerically and experimentally by a number of investigators (see, for example, the works by Nandakumar and his co-workers and Finlay and his co-workers), the symmetric solution (in particular, the four cell flow) can be stable in some parameter region but unstable to various perturbations in some other parameter range for the Dean- , Coriolis-, mixed convection- and combined-problems, leading the flow to be asymmetric or in the form of travelling waves. A thoroughly numerical calculation should allow the flow to be asymmetric and carry out secondary and higher order instability analyses w.r.t. various perturbations. Such analyses are beyond the scope of the present study and are believed to be difficult for a pressure-driven flow in a channel with finite span and simultaneous effect of the curvature, the rotation and the heating/cooling. However, even in the range of the dynamical parameters where the instabilities could essentially cause the flow to be asymmetric, a symmetric analysis appears still worthy to carry out in the sense that (1) the flow from such analyses forms the base flow which one must know in order to make instability analyses, and that (2) such an analysis would indicate where in the dynamical parameter space one should make the higher order instability analyses. 


\section{NUMERICAL METHOD OF SOLUTION}

The governing equations (1)-(5) are a set of convectiondiffusion equations with velocity-pressure coupling. In order to obtain solution for this kind of equations by finite-volume method, two factors are considered to be extremely essential: (1) using the correct difference scheme for convection term and (2) decoupling the velocity and pressure properly. After discretization in the domain, the governing differential equations become a set of algebraic equations, the so-called discretization equations. The methods of solving these discretization equations are also vital to the success. Therefore the difference scheme, treatment of the velocity-pressure coupling and the method of solving the discretization equations may be regarded as three major factors for the success of a finite-volume method. And they are also the major criteria for distinguishing one scheme from the other.

In the last twenty years, numerous papers were published dealing with the three aspects of the finite-volume method mentioned above. Based on the review and comparison among various methods in terms of their transport and conservative properties, convective numerical stability, economy and exactitude, we chose the power-law scheme to discretize the convection term; employ the SIMPLE scheme to deal with the problem of velocity-pressure coupling; and use an alternating direction line-by-line iterative method (ADI) with block correction technique to solve the discretization equations. The description of the numerical implementation can be found, for example, in Patankar. ${ }^{24}$

The initial calculation for the Dean problem was performed by setting angular velocity $\Omega=0$ to verify the code. In Fig. 2, the mean friction factor and Nusselt number for curved square channel obtained by the present analysis are shown together with the available theoretical, numerical and experimental results. In the figure, the friction factor and Nusselt number are shown as a function of the Dean number on the basis of those for a stationary straight square channel. The results of the present analysis are in good agreement with the published results.

In the present computations, four pairs of grid sizes uniformly distributed in the flow domain were used to check the grid dependence. They are $35 \times 17,43 \times 21,51 \times 25$ and $59 \times 29$. The results obtained by using these four grid sizes are shown in Table I for six cases at $L_{1}=-30,-5,1,5,8$ and 30 , respectively, with $\gamma=1, \sigma=0.01, \operatorname{Pr}=0.7, D k=100$ and $L_{2}=-5$. These six cases are chosen because they cover all typical secondary flow patterns obtained in the present work. Four representative properties, namely, the Dean number ( $D e=\operatorname{Re} \sqrt{\sigma}, R e=W_{m} d_{h} / \nu$ with $W_{m}$ as the axial mean velocity), the maximum of absolute values of secondary flow stream function $\left(|\psi|_{\max }\right)$, maximum axial velocity $\left(w_{\max }\right)$ and maximum temperature $\left(t_{\max }\right)$, as well as the CPU time, are listed in Table I for comparison. ${ }^{33}$ The computations were carried out on the AMDAHL computer. The initial guesses of the fields for $u, v, w, t$, and $p$ were all set to zero. The general trend of these results as the grid size is decreased tends to indicate that the solutions for the case of $(51 \times 25)$ grids are accurate to within $1 \%$ tolerance. We also checked the detailed variations of the flow and temperature
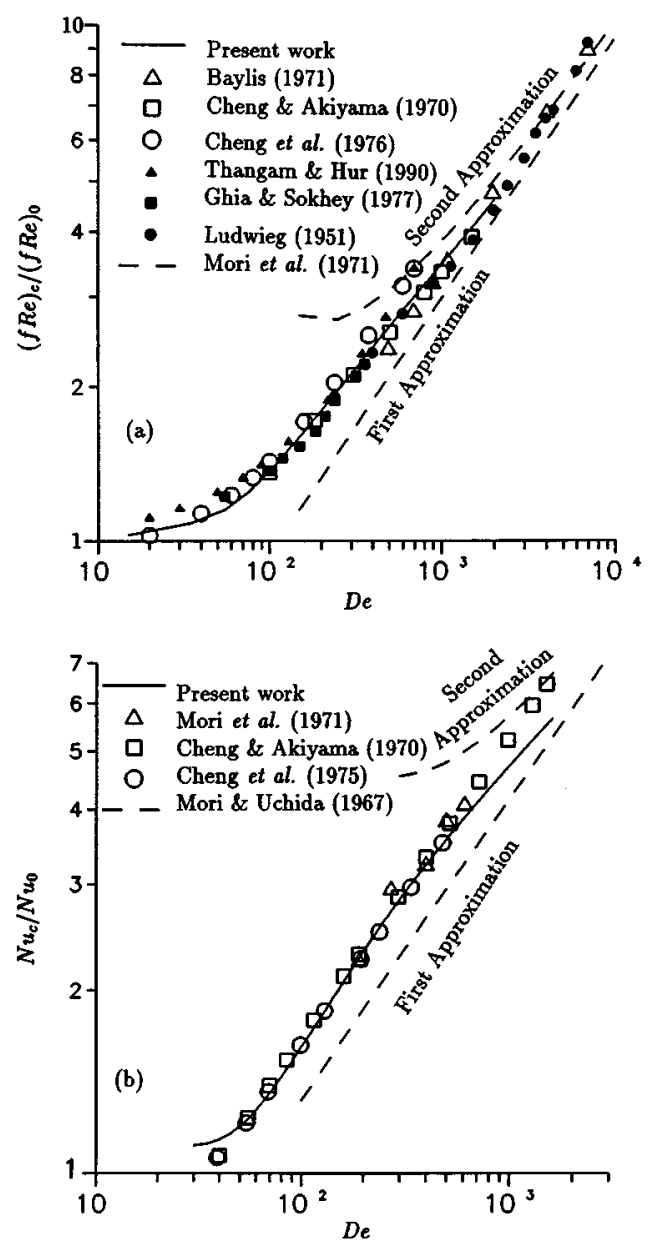

FIG. 2. Variations of friction factor and Nusselt number with Dean number for a stationary square channel-a comparison of the present results with available theoretical, experimental and numerical findings: (a) friction factor (Baylis $1971^{25}$ : experiments; Cheng and Akiyama $1970^{26}$ : numerical analysis; Cheng et al. 1976 ${ }^{27}$ : numerical analysis; Thangam and Hur $1990^{28}$ : numerical analysis; Ghia and Sokhey $1977^{29}$ : numerical analysis; Ludwieg $1951^{13}$ : Experiments; Mori et al. $1971^{30}$ : boundary layer correlations); (b) Nusselt number (Mori et al. 1971 ${ }^{30}$ : experiments; Cheng and Akiyama $1970^{26}$ : numerical analysis; Cheng et al. $1975^{31}$ : numerical analysis; Mori and Uchida $1967^{32}$ : boundary layer correlations).

fields for different grid sizes, and found that $51 \times 25$ is indeed a reasonably accurate choice for the grid size for square channels. It is worth noting that the CPU time increases rapidly as the grid spacing decreases. In order to have a balance between the cost of the computer time and the accuracy of the solution, we carried out all the computations with a $51 \times 25$ uniform meshes for square channels. ${ }^{34}$

Typically, the computations were made for given values of the aspect ratio $\gamma$, curvature ratio $\sigma$, Prandtl number $\operatorname{Pr}$, Pseudo Dean number $D k$ and the parameters $L_{1}$ and $L_{2}$. The calculations were performed iteratively using the alternating direction line-solution (ADI) with the block correction technique, and the solution was assumed to be convergent in a numerical sense if the maximum relative error in each of the primitive variables (i.e., velocity components, temperature and the pressure) is less than $5 \times 10^{-5}$ between successive iterations. 
TABLE I. Variations of $D e,|\psi|_{\max }, w_{\max }, t_{\max }$, and CPU time in seconds with different grids.

\begin{tabular}{|c|c|c|c|c|c|c|}
\hline$L_{1}$ & $\begin{array}{l}\gamma=1 \\
\text { Grids }\end{array}$ & $\begin{array}{c}D k=100 \\
D e\end{array}$ & $\begin{array}{c}\sigma=0.01 \\
|\psi|_{\max }\end{array}$ & $\begin{array}{c}P r=0.7 \\
w_{\max }\end{array}$ & $\begin{array}{c}L_{2}=-5 \\
t_{\max }\end{array}$ & CPU (s) \\
\hline \multirow[t]{4}{*}{-30} & $35 \times 17$ & 46.93 & 12.86 & 0.0177 & 16.79 & \\
\hline & $43 \times 21$ & 47.94 & 12.88 & 0.0182 & 17.22 & 86.5 \\
\hline & $51 \times 25$ & 48.66 & 12.88 & 0.0184 & 17.54 & 184.4 \\
\hline & $59 \times 29$ & 49.04 & 12.88 & 0.0185 & 17.66 & 465.7 \\
\hline \multirow{4}{*}{-5} & $35 \times 17$ & 63.33 & 13.47 & 0.0252 & 23.35 & \\
\hline & $43 \times 21$ & 64.02 & 13.38 & 0.0273 & 26.75 & 125.7 \\
\hline & $51 \times 25$ & 68.22 & 13.51 & 0.0286 & 28.61 & 492.7 \\
\hline & $59 \times 29$ & 68.50 & 13.47 & 0.0286 & 28.85 & 1300.8 \\
\hline \multirow[t]{4}{*}{1} & $35 \times 17$ & 72.30 & 11.13 & 0.0299 & 29.85 & \\
\hline & $43 \times 21$ & 72.75 & 11.07 & 0.0301 & 30.19 & 170.5 \\
\hline & $51 \times 25$ & 73.01 & 11.03 & 0.0302 & 30.38 & 370.4 \\
\hline & $59 \times 29$ & 73.17 & 11.04 & 0.0302 & 30.49 & 750.9 \\
\hline \multirow[t]{4}{*}{5} & $35 \times 17$ & 90.11 & 10.57 & 0.0405 & 49.37 & \\
\hline & $43 \times 21$ & 90.45 & 10.64 & 0.0407 & 49.79 & 106.5 \\
\hline & $51 \times 25$ & 90.63 & 10.63 & 0.0407 & 49.95 & 214.0 \\
\hline & $59 \times 29$ & 90.74 & 10.65 & 0.0408 & 50.03 & 369.0 \\
\hline \multirow[t]{4}{*}{8} & $35 \times 17$ & 101.29 & 9.32 & 0.0464 & 66.76 & \\
\hline & $43 \times 21$ & 101.45 & 9.43 & 0.0465 & 66.71 & 162.0 \\
\hline & $51 \times 25$ & 101.54 & 9.51 & 0.0464 & 66.83 & 282.5 \\
\hline & $59 \times 29$ & 101.60 & 9.54 & 0.0464 & 66.81 & 1540.3 \\
\hline \multirow[t]{4}{*}{30} & $35 \times 17$ & 51.83 & 10.37 & 0.0194 & 21.24 & \\
\hline & $43 \times 21$ & 52.81 & 10.33 & 0.0198 & 21.80 & 79.6 \\
\hline & $51 \times 25$ & 53.50 & 10.29 & 0.0201 & 22.13 & 180.0 \\
\hline & $59 \times 29$ & 53.97 & 10.26 & 0.0202 & 22.28 & 427.1 \\
\hline
\end{tabular}

\section{RESULTS AND DISCUSSION}

In addition to the usual viscous and inertial forces, the fluid in the rotating curved channel is subjected to centrifugal force (due to the curvature of the channel), Coriolis force (due to the rotation and curvature) and the centrifugal-type buoyancy force (resulting from temperature-induced density variation of the fluid in the rotating field). While the centrifugal and buoyancy forces act on the plane of cross section, the Coriolis forces have both $X$ and $\theta$ components for the configuration, as shown in Fig. 1. That due to the curvature is $-U W /\left(R_{c}-a / 2+X\right)(\theta$-component). Those due to rotation are perpendicular to both the axis of rotation and the direction of the relative velocity of the fluid, i.e., $-2 \Omega U$ $(\theta$-component $)$ and $2 \Omega W \quad(X$-component $) . \quad$ Two $\theta$-components of the Coriolis force may act in the direction or direction opposite to the main flow depending on the signs of $U$ and $\Omega U$. Consequently, they may accelerate or decelerate the main flow. Furthermore, these two components either enhance or cancel each other depending on the rotation direction of the channel. If the rotation is positive, they enhance each other. Otherwise, they cancel each other.

In the plane of the cross section, the centrifugal force always acts outwards in the positive $X$-direction. However, the Coriolis force may act in either the positive or negative $X$-direction depending on the rotation direction. If the rotation is positive, it will act along the positive $X$-direction. When the rotation is negative, however, it will act in the negative $X$-direction. Similarly, the centrifugal-type buoyancy force may act in the positive or negative $X$-direction depending on the direction of the heat flux. If the fluid is heated, it will be along the positive $X$-direction. If fluid is cooled, it will act in the negative $X$-direction. From this simple analysis about force directions, it is clear that centrifugal, Coriolis and buoyancy forces enhance each other for some cases, and cancel each other for other cases. This would make the flow and heat transfer more complex than those of channel with simple rotation or curvature.

The flow and heat transfer under consideration are characterized by six dimensionless parameters: $\gamma, \sigma, \operatorname{Pr}, D k, L_{1}$ and $L_{2}$. These parameters are coupled with and affect each other, so that the effects of the parameters on the flow and heat transfer are very complex. Extensive computations are required for a large number of cases to cover the entire effect, which requires an extremely long computation time. Fortunately, the introduction of $L_{1}$ and $L_{2}$, analogous to the normalization of the problems, enables one to obtain some insight of the problem from not so large a number of cases. This results from the fact that they are the dynamical parameters for the Coriolis problem and mixed convection problem based on the dynamical parameter for the Dean problem. ${ }^{3,14,23}$ Even so, however, it is still a lengthy process to describe the typical results covering the whole range of the parameters. The results shown in this paper will be mainly confined to the case of the positive rotation with $\gamma=1, \sigma=0.02$ and $\operatorname{Pr}=0.7$ with the exception of those in Sec. IV B. The readers are referred to $\mathrm{Wang}^{3}$ for the case of negative rotation.

Some features of the main flow and temperature distributions can be expected and understood through the force balance and energy balance in the governing equations. It is the secondary flow that makes the axial velocity and temperature profiles different from the parabolic profile in Poiseuille flow. The effect of the secondary flow enters the governing equation for the main flow [Eq. (4)] through three terms: the convection term, and two Coriolis terms due to the curvature and rotation, respectively. Two Coriolis terms may be in the same direction or opposite to the main flow depending on the sign of $U$ although they are always in the same direction for the case of positive rotation. The absence of these three terms leads to the Poiseuille solution which has an axisymmetric and parabolic profile. If $D k, L_{1}$ and $L_{2}$ are small enough, then secondary flow would be too weak to modify the main flow and temperature distributions effectively. Such axial velocity and temperature profiles are essentially axisymmetric and parabolic with the maximum value occurring along the horizontal centerline at or very close to the center of the cross section. One case with this kind of flow and temperature distribution is shown in Fig. 3. This is a limiting case examined by previous studies. ${ }^{16} \mathrm{In}$ this flow region, the inertial force in Eq. (4) is very weak as compared with the viscous force. The driving force for main flow (i.e., pressure term) is mainly balanced by the viscous force in whole flow domain. Other forces (inertial, Coriolis forces) are very weak. The stability analysis, performed by Winters ${ }^{5}$ and Yanase, Goto and Yamamoto, ${ }^{35}$ showed that this one-pair vortex flow is stable to an arbitrary perturbation in the Dean problem.

The effect of the secondary flow enters the energy equation through one term only, i.e., convection term. When the secondary flow is sufficiently weak such that the Coriolis 

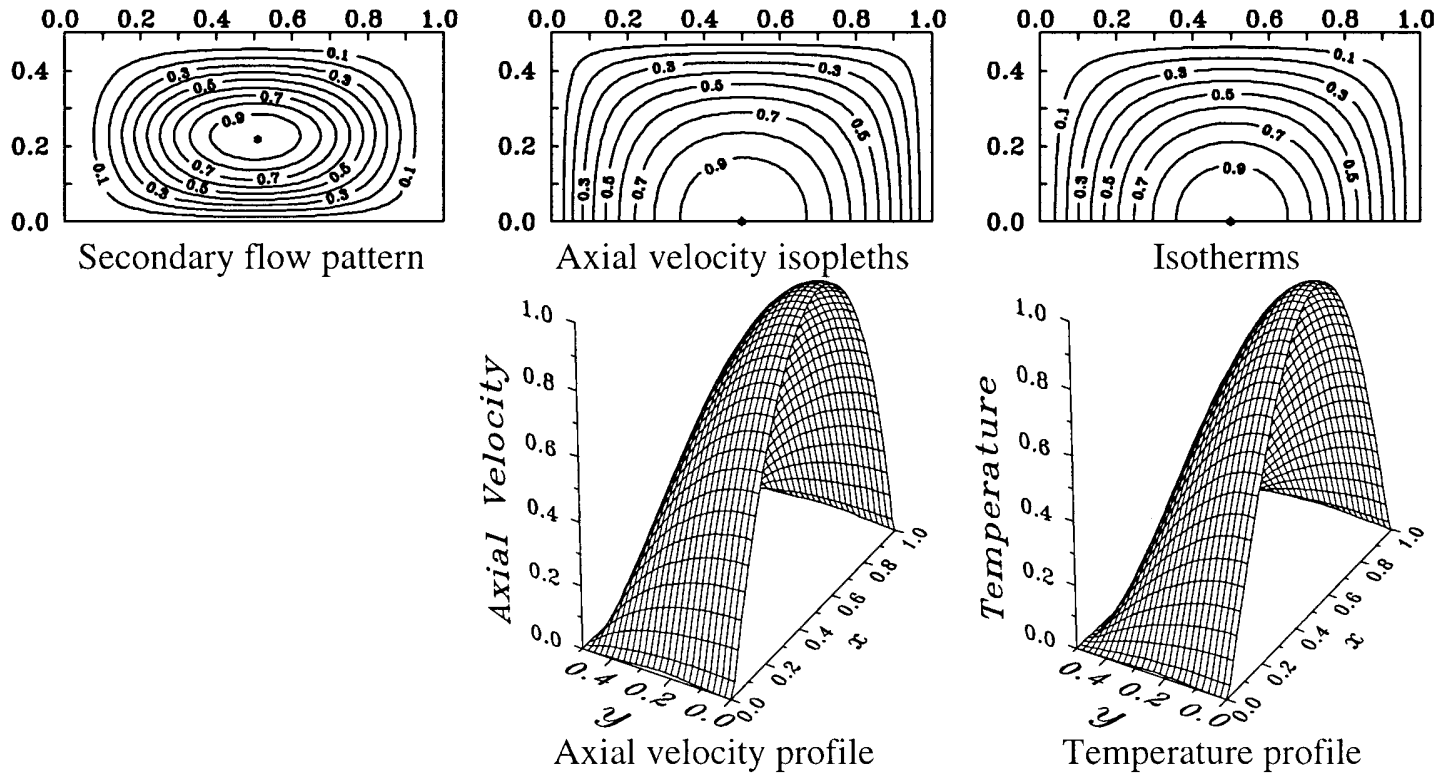

FIG. 3. Weak curvature and rotation ( $\gamma=1, \sigma=0.02, P r=0.7, D k=5, L_{1}=0.01, L_{2}=0, R e=35, R o=0.000952, R a_{\Omega}=0$. Maxima of stream function, axial velocity and temperature are $0.0592,0.0737$ and 4.064 , respectively).

terms in the momentum equation in the $\theta$-direction are too weak to modify the main flow effectively, the main flow and temperature distributions should be qualitatively similar. However, they might have qualitatively different distributions if the secondary flow is strong enough.

\section{A. Flow transitions and temperature distributions}

\section{The case without the effect of buoyancy force} $\left(L_{2}=0\right)$

Figure 4 illustrates the secondary flow patterns, axial velocity isopleths and profiles, and isotherms and temperature profiles for several representative values of $L_{1}$ at $\sigma=0.02, D k=100$ and $L_{2}=0$. Because of the symmetry about the horizontal centerline, they are shown in the upper half of the cross section only. In the figure, the stream function, axial velocity and temperature are normalized by their corresponding maximum absolute values $|\psi|_{\max }, w_{\max }$ and $t_{\max }$. A star is used to denote the position at which they reach the maximum values. A vortex with a positive (negative) value of the stream function indicates a counterclockwise (clockwise) circulation.

With zero value of $L_{2}$, centrifugal-type buoyancy force disappears. Heat transfer is purely forced convection. In the plane of cross section, both centrifugal (due to curvature) and Coriolis forces (due to the rotation) act radially outwards $(+X)$ for the case of positive rotation. If $L_{1}$ is also set to zero, the Coriolis force then disappears and the problem reduces to the classical Dean problem which has been well examined by many investigators. The secondary flow consists of one-pair of counter-rotating vortices as shown in Fig. 4(a)-(i) for the case of low Dean number. The fluid in the core region is driven in the positive $X$-direction by the centrifugal force. The outward flow in the core region forces the fluid near the upper and lower walls to flow in the negative $X$-direction and one-pair of counter-rotating vortices is generated. These are the so-called Ekman-vortices. The strong inward flow near the upper and lower wall is observed and this induces the Ekman layer ${ }^{36}$ [Fig. 4(a)-(ii)]. A uniform outward secondary flow in the core region has two effects on the main flow. One is pushing the axial velocity peak outward, thereby increasing the local shear stress and heat transfer near the outer wall. Another is inducing an upstream Coriolis force, which flattens the axial velocity profile [Fig. 3(a)-(ii)].

As $L_{1}$ increases from zero, the secondary flow becomes stronger since the outward Coriolis force enhances the centrifugal force. However, it consists of the same type of one pair of counter-rotating vortices, as shown in Fig. 4(b)-(i). There exists a weak secondary flow region in triangular form [Fig. 4(b)-(i)] near the central part of the outer wall. This foreshadows the onset of an Coriolis instability to be described later.

Shown in Figs. 4(a), (b)-(ii) are the axial velocity isopleths and profiles corresponding to the secondary flow patterns in Figs. 4(a), (b)-(i). It is observed that the isovels are more sparsely spaced in the region near the inner wall than near the outer wall. Consequently, pronounced peripheral variations are expected in the local friction factors. The densely distributed isovels near the center part of the outer wall results in a high pressure region, since centrifugal force and Coriolis force are proportional to $W^{2}$ and $W$, respectively. The flow in the channel core is not geostrophic; it is ageostrophic, i.e., pressure gradients are balanced by both Coriolis force and convective inertial force. Two axial velocity peaks are observed with one on the upper half of the cross section and the other on the lower half. The regions of maximum velocity are moved toward the upper and lower walls while they are shifted toward the outer wall by the curvature and rotation in the positive direction. There is an indication of peaking of the axial velocity near the boundary layer re- 

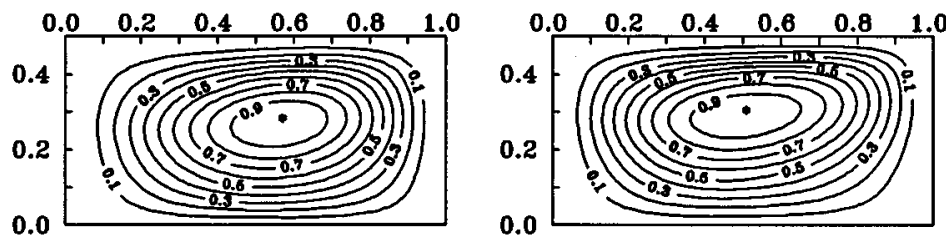

(i) Secondary flow patterns
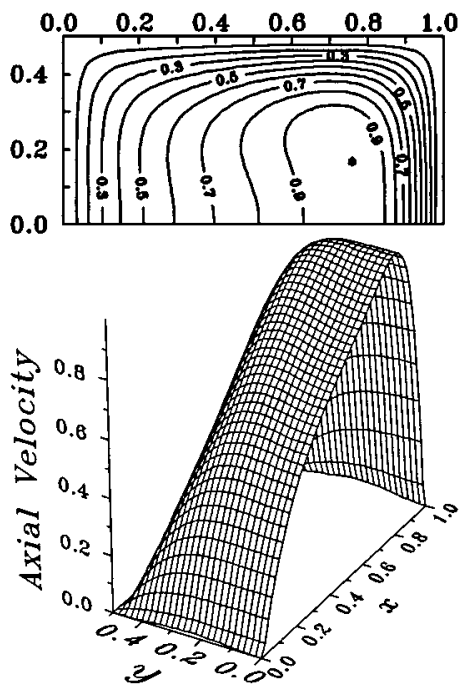
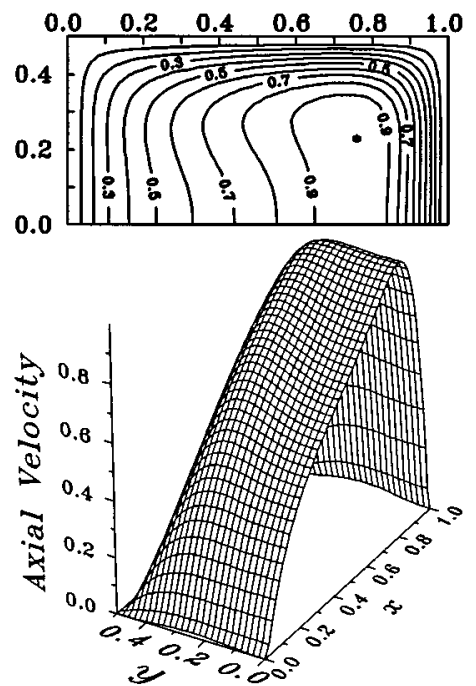
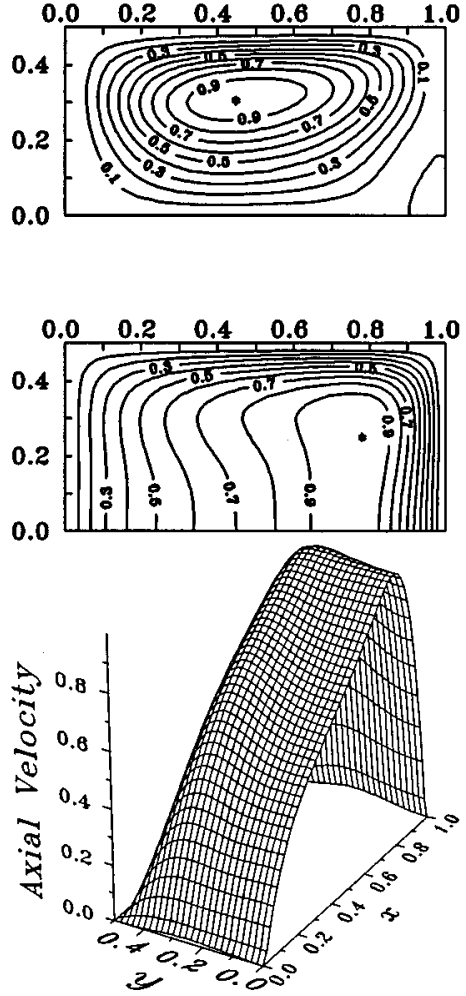

(ii) Axial velocity isopleths and profiles
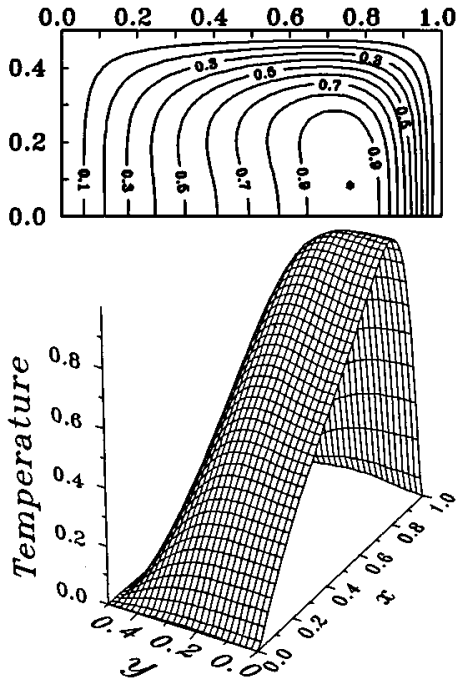
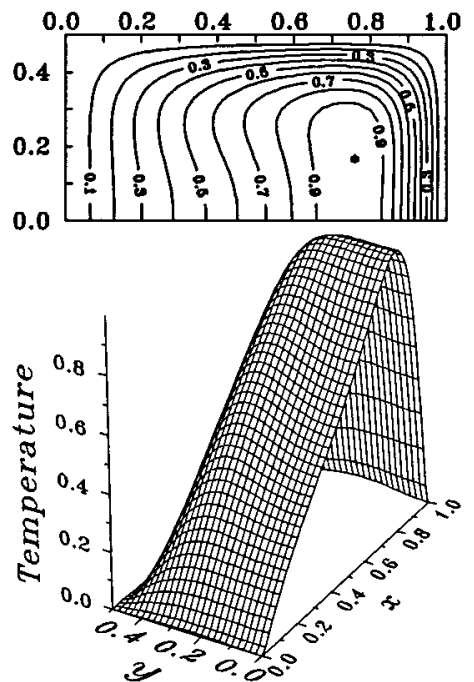
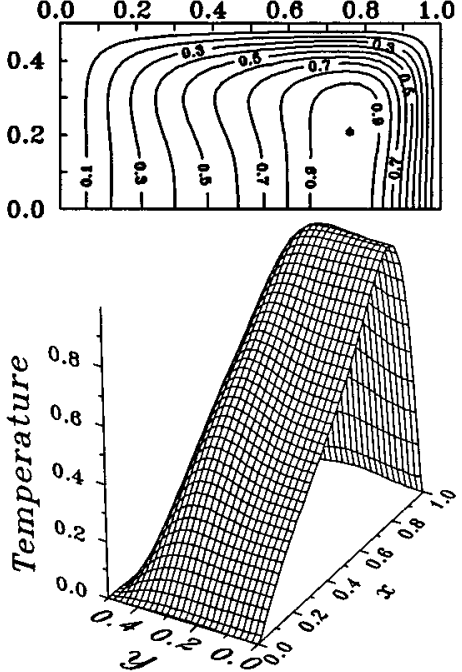

(iii) Isotherms and temperature profiles
(a) $L_{1}=0 ; \operatorname{Re}=542 ; R o=0$
(b) $L_{1}=1 ; \operatorname{Re}=494 ; R o=0.135$
(c) $L_{1}=2.5 ; \operatorname{Re}=453 ; R o=0.368$
$(5.641 ; 0.0496 ; 39.89)$
$(6.865 ; 0.0440 ; 31.88)$
$(7.778 ; 0.0394 ; 26.65)$

FIG. 4. Flow transitions and temperature distributions at $\gamma=1, \sigma=0.02, \operatorname{Pr}=0.7, D k=100$ and $L_{2}=0\left(R a_{\Omega}=0\right)$. [Three values for each case are the maxima of the absolute value of the stream function $\left(|\psi|_{\max }\right)$, axial velocity $\left(w_{\max }\right)$ and temperature $\left.\left(t_{\max }\right).\right]$

gions at the upper and lower walls. The peaking results because the boundary layer is being fed by high velocity fluid from the outer wall; the core, however, is being fed by lower velocity fluid from the inner wall. A depression in the axial velocity profile near the outer wall shown in Fig. 4(b)-(ii) foreshadows the onset of the instability to be described later.

A striking feature of this ageostrophic one-pair vortex structure can be seen from the profiles of the axial velocity along the vertical centerline and the horizontal centerline by curves 0 and 1 in Fig. 5. The axial velocity $w$ changes linearly across the core and slightly from the lower to the upper wall. Then the vorticity is nearly constant. Slow-moving fluid from the inner wall is accelerated across the core axially at a constant rate until the outer wall is approached. Coriolis 

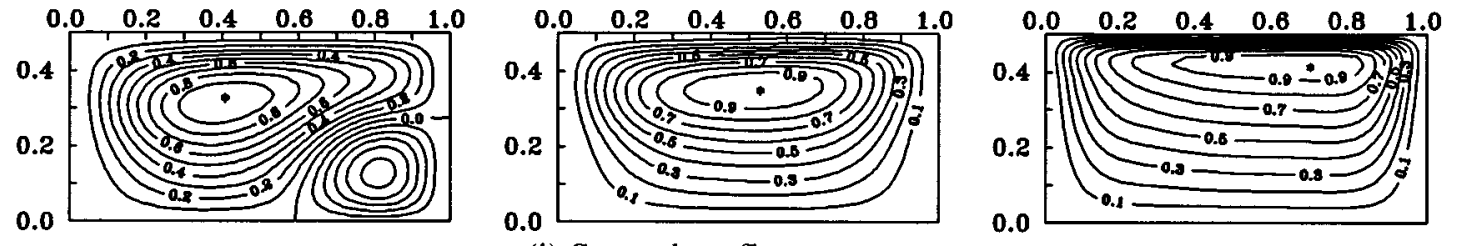

(i) Secondary flow patterns
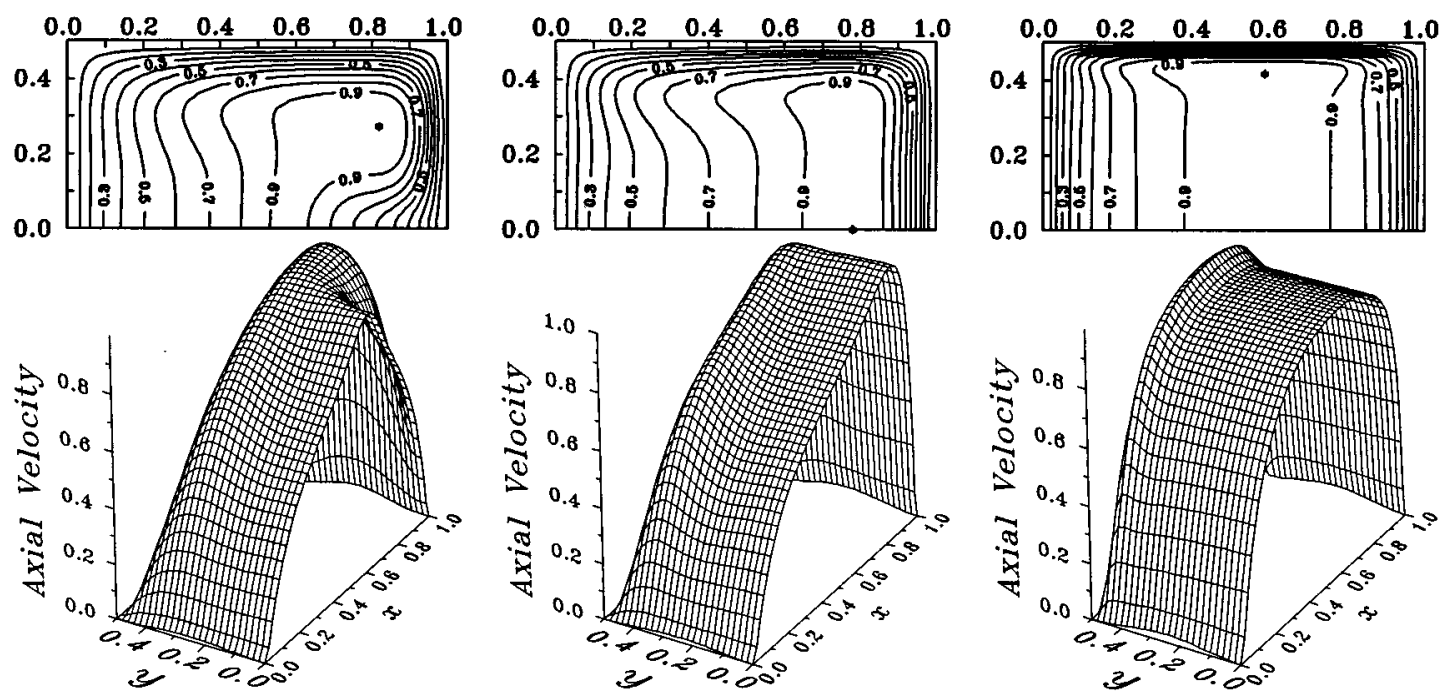

(ii) Axial velocity isopleths and profiles
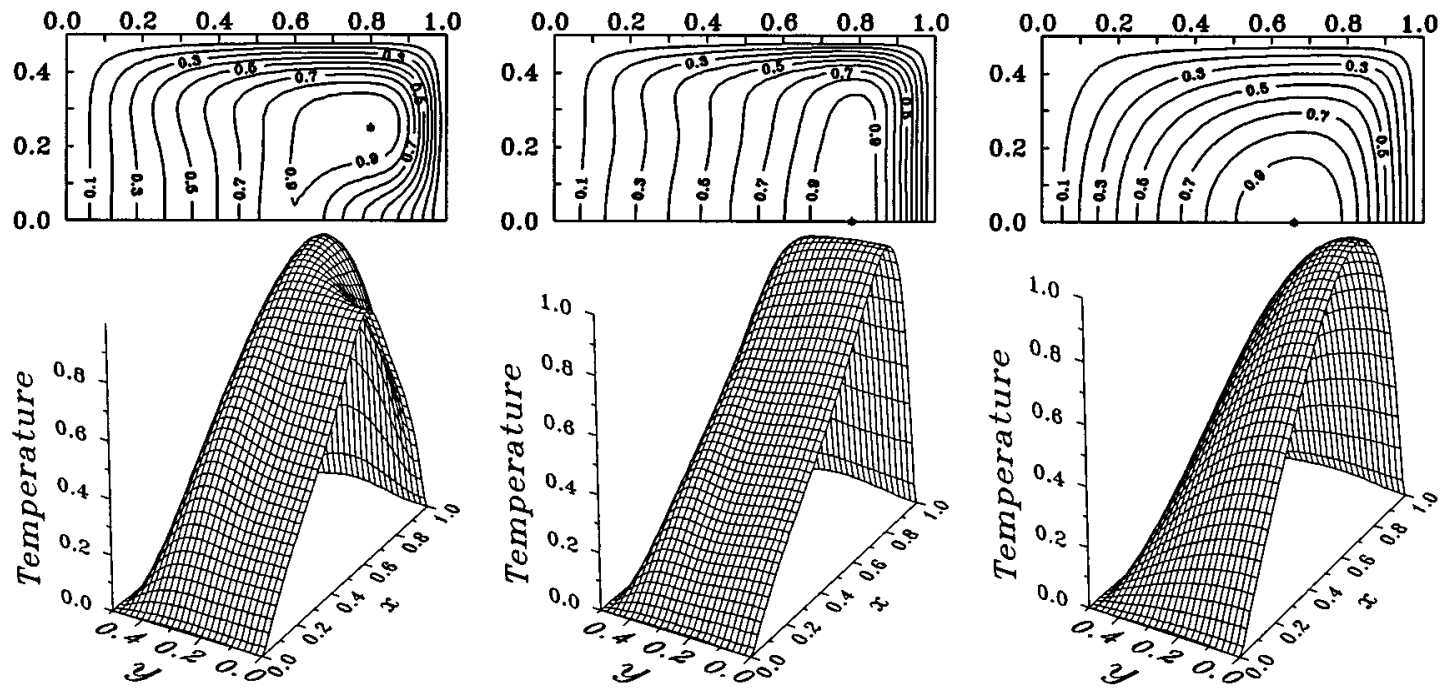

(iii) Isotherms and temperature profiles

(d) $\begin{aligned} L_{1}=3 ; \mathrm{Re}=423 ; R o=0.473 \\ (7.256 ; 0.0353 ; 22.40)\end{aligned}$ (e) $L_{1}=10 ; \operatorname{Re}=363 ; R o=1.837$

$(9.239 ; 0.0293 ; 19.16)$ (f) $L_{1}=70 ; \mathrm{Re}=195 ; R o=23.93$

$(3.606 ; 0.0132 ; 15.06)$

FIG. 4. (Continued.)

and convective inertial forces dominate the ageostrophic core. Axial velocity falls to zero in the viscous layers on the inner and outer walls. The constant-vorticity, inviscid core flow structure suggests a possible analysis by the asymptotic method. It appears that no such attempt was made so far in the literature.

Qualitatively similar results are observed for the temperature distribution [Figs. 4(a),(b)-(iii)]. The pronounced peripheral variations in the local Nusselt number will result from the more sparsely spaced isotherms in the region near the inner wall than near the outer wall. The reason for this is that the larger axial velocity gradients exist in the region near the outer wall. Two symmetric (with respect to the horizontal centerline) high temperature regions are observed. The physical mechanism can be explained by recalling the secondary flow patterns in Figs. 4(a),(b)-(i). The larger inward 

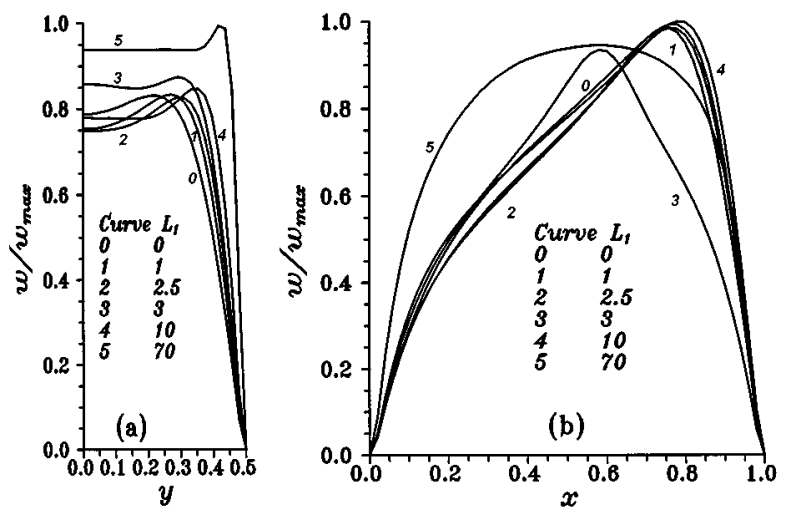

FIG. 5. Axial velocity distributions at $\gamma=1, \sigma=0.02, \operatorname{Pr}=0.7, D k=100$ and $L_{2}=0\left(R a_{\Omega}=0\right)$ : (a) along the vertical centerline of the channel; (b) along the horizontal centerline of the channel.

secondary flow near the upper and lower walls brings the relatively cold (i.e., non-dimensional temperature close to zero) fluid to the inner wall; the colder fluid near the inner wall returns to the core region of the channel, flows outward and isolates the warmer fluid in the upper and lower sides of the channel. And the fairly uniform outward secondary flow in the core region leads to a flattened isotherm distribution in that region.

When the rotation becomes more rapid, the ageostrophic one-pair vortex breaks down into a configuration of two-pair of counter-rotating vortices that is asymmetric with respect to the vertical centerline of the channel [Figs. 4(c),(d)-(i)]. The additional pair of counter-rotating vortices located in the center of the outer wall are called Coriolis-vortices. They result from the Coriolis instability and are similar to the Dean-vortices due to the centrifugal instability. A strong inward secondary flow exists between these two vortices and a strong outward secondary flow appears between the original larger vortices (Ekman-type-vortices) and the smaller vortices (Coriolis-vortices).

The onset of the Coriolis-vortices is consistent with the instability explanation given by Cheng et al. ${ }^{27}$ for the Dean problem. In the region near the central outer wall, the pressure gradient across the channel in the $X$-direction is positive, but the centrifugal force and Coriolis force decrease from a maximum value to zero at the outer wall. The instability due to the imbalance between the pressure gradient inwards and the Coriolis and centrifugal forces outwards results in an unstable region. If the rotation speed becomes large enough, viscous effects can no longer hold the one-pair vortex structure in place, thus additional vortices may appear. The resulting secondary flow is similar to those obtained by Cheng et al. ${ }^{27}$ for the Dean problem and Speziale ${ }^{37}$ for the Coriolis problem. However, the instability here is caused by both the Coriolis and centrifugal forces rather than by centrifugal force (Coriolis force) alone as in the Dean problem (Coriolis problem). Since no such instability can exist in the absence of the Coriolis force [Fig. 4(a)-(i)], we may still call this instability the Coriolis instability, and the resulting additional pair of vortices as the Coriolis-vortices.

Figures 4(c),(d)-(ii) demonstrates the way in which the
Coriolis-vortices affect the isovels and the profile of the axial velocity. Significantly distorted isovels are observed in the region with the Coriolis-vortices. Corresponding to the strong inward secondary flow, the isovels in that region are moved inwards by the Coriolis-vortices. Two symmetric high velocity cores are found. The position of the maximum axial velocity, at which the centrifugal and Coriolis forces are maximum, is located on the boundary line between the Ekman and Coriolis-vortices. It is clear that the centrifugal and Coriolis forces due to the main flow become the driving forces for the secondary flow. Comparing with those in Figs. 4(a),(b)-(ii), the isovels in the region near the upper and lower wall are more tightly spaced. The large velocity gradients are also found between each of the two high velocity cores and the outer wall. Thus the higher local friction factors are expected there.

The axial velocity distributions along the vertical and horizontal centerlines are shown by curves 2 and 3 in Figs. 5(a),(b). Again, the axial velocity is substantially distorted, with its maximum velocity shifting toward the low-pressure side of the channel [Fig. 5(b)]. The axial velocity profile along the vertical centerline is symmetric and flat with peaks located near the boundary of the boundary layers at the upper and lower walls. The most striking feature is that the axial velocity has inflection points on both the vertical and horizontal centerlines. Similar features were found in the Dean problem ${ }^{27}$ and Coriolis problem. ${ }^{37}$ From inviscid reasoning, such profiles may be unstable in accordance with Rayleigh's inflection point criterion. Assuming the channel with infinite span, the linear stabilities of Dean-vortices (in Dean problem) and Coriolis-vortices (in Coriolis problem) were examined by Finlay, Keller and Ferziger ${ }^{38}$ and Finlay. ${ }^{39}$ Two different wavy travelling vortex flows, namely undulating vortex flow and twisting vortex flow, are developed due to the instability of the Dean-vortices and Coriolis-vortices subjected to the streamwise wavy perturbations. These two kinds of vortex flows are confirmed experimentally by Ligrani et al. ${ }^{40}$ and Ligrani and Niver ${ }^{41}$ for the Dean problem with large aspect ratio.

The stability of Dean-vortices and Coriolis-vortices subjected to two-dimensional, spanwise-periodic perturbations (i.e., Eckhaus stability) was examined numerically by Guo and Finlay ${ }^{42}$ for infinite-span cross section. They found that Eckhaus stability boundary is a small closed loop. Within the boundary, Dean-vortices or Coriolis-vortices are stable to spanwise perturbations. Outside the boundary, Eckhaus instability causes the vortex pairs to split apart or merge together. Experimental observations of splitting and merging of vortex pairs were made by Ligrani and Niver, ${ }^{41}$ Alfredsson and Persson ${ }^{43}$ and Matsson and Alfredsson. ${ }^{44}$ However, this type of hydrodynamic stability analysis has not been extended to the geometries with finite cross sections. It is also noted that such an analysis has not been made for buoyancy-vortices which will be discussed later.

Based on a displaced particle argument, ${ }^{45}$ a stability criterion was derived in Wang ${ }^{3}$ and Wang and Cheng ${ }^{46,47}$ to analyze stable and unstable regions of flows with respect to the primary instability in a rotating curved channel of an infinite span. Such an analysis did not include the effect of 
the buoyancy force, and has not been extended to the case with a finite channel span in which the base flow is more complex. For a channel with a positive rotation and a small curvature ratio $\sigma$, the flow was found to be stable in a region near the inner wall and unstable in a region near the outer wall when $R o \leqslant 3$ ( $R o$ is the rotation number defined as $R o=\Omega a / W_{m}, a$ is the gap width of the channel). As well the unstable region reduces as $R o$ increases, and the high rotation with $R o \geqslant 3$ always stabilizes the flow in the whole cross section. However, the flow visualization showed that such an analysis is only valid when the rotation is very weak $(R o<<1)^{3,46,47}$ The evidence of possible secondary instabilities revealed in the present work provides an explanation that the flows may be controlled by the secondary instabilities rather than the primary instability.

Figures 4(c),(d)-(iii) show that isotherms and temperature profile are affected by the Coriolis-vortices. The isotherms are drastically distorted inward near the center of the outer wall where the Coriolis-vortices occur. Two symmetric high temperature cores (with respect to the horizonal centerline) appear in the cross section of the channel. The isotherms in the regions near the upper and lower walls are more tightly spaced than those shown in Figs. 4(a),(b)-(iii). Larger temperature gradients are also found between each of the two high temperature cores and the outer wall. Thus a higher heat transfer rate is expected in these regions.

If the Coriolis force is now increased to that with $L_{1}=10$ while maintaining the same values for the other parameters, the Coriolis vortex pair presented in Figs. 4(c),(d)(i) disappears and the secondary flow restabilizes to a slightly asymmetric one-pair vortex configuration, as shown in [Fig. 4(e)-(i)]. Furthermore, the inflection points in the axial velocity profiles along the vertical and horizontal centerlines also disappear (curve 4 in Fig. 5). The axial velocity profile assumes a Taylor-Proudman configuration in the core region with a maximum located on the horizontal centerline [Fig. 4(e)-(ii)]. The tightly spaced isovels along the outer wall, upper and lower walls signal the high local friction factors in these regions. The similarity between the axial velocity and temperature profiles still holds although the difference between them becomes larger than the previous cases.

After the Coriolis-vortices disappear, there still exists a transition in the main flow upon increasing the Coriolis force further. If the value of $L_{1}$ is high enough, as shown in Fig. 4(f)-(ii), the Coriolis forces tend to dimple the axial velocity profile in the region near the center and create a dumbbelllike profile with two maxima. One is within the Ekman layer along the upper wall. The other is within the Ekman layer along the lower wall. The shifting of the locations of maxima results in even more closely spaced isovels near the upper and lower walls. The fluids flow geostrophically in the channel core and the Stewartson layers (vertical double layers). However, the secondary flow and temperature profile [Fig. 4(f)-(i),(iii)] remain qualitatively similar as those shown in Fig. 4(e)-(i),(iii). It appears that this is the first numerical calculation to illustrate two kinds of flow structures after the Coriolis-vortices disappear.

It is worthy to note that near uniformity of the axial velocity in the core region of the rotating curved channels is of great importance in aerosol centrifuges. It allows aerosol centrifuges to function as true particle spectrometers. ${ }^{48,49}$

\section{The heating case with $L_{2}>0$}

If the fluid is heated, both centrifugal and centrifugaltype buoyancy forces act radially outwards in the plane of the cross section. The Coriolis force also acts radially outwards in the case of positive rotation. The flow transitions in both secondary flow and main flow are qualitatively similar to those for the case of $L_{2}=0$. However, the corresponding transitions will occur at lower values of $L_{1}$ than those for $L_{2}=0$. The difference results from the enhancement effect of the buoyancy force on Coriolis force and centrifugal forces, and depends on the relative importance of the buoyancy force, i.e., the value of $L_{2}$.

Figure 6 shows the secondary flow patterns, axial velocity isopleths and profiles, and isotherms and temperature profiles for three typical values of $L_{1}$ at $\sigma=0.02, D k=100$ and $L_{2}=5$. The axial velocity profiles, along the vertical and horizontal centerlines, are illustrated in Fig. 7.

Shown in Fig. 6(a)-(i) is one secondary flow with an additional pair of vortices occurring in the center part near the outer wall. A comparison with Figs. 4(a),(b)-(i) indicates that they result from the buoyancy force instability [note that no such vortices can exist in the absence of the buoyancy force, Figs. 4(a),(b)-(i)], and are called buoyancy-vortices which are similar to the Dean-vortices due to the centrifugal instability and Coriolis-vortices due to the Coriolis instability. The presence of the buoyancy-vortices leads to a highly disturbed main flow field [Fig. 6(a)-(ii)] and temperature field [Fig. 6(a)-(iii)], with strong inflectional profiles developing on both vertical and horizontal centerlines (curve 1 in Fig. 7). This can result in a secondary instability which demand further investigation in the future. The secondary flow, the main velocity and temperature distributions (Figs. 6(a)(i),(ii),(iii)] are qualitatively similar to those with the Coriolis vortices, as shown in Figs. 4(d)-(i),(ii),(iii).

Upon increasing the rotation speed to $L_{1}=5$, the buoyancy vortex pair in Fig. 6(a)-(i) disappears, and the secondary flow reduces to one pair counter-rotating configuration [Fig. 6(b)-(i)]. Also disappeared are the inflection points in the axial velocity profiles along the vertical and horizontal centerlines (curve 2 in Fig. 7). This is similar to the disappearance of the Coriolis vortices as increasing the rotation speed [Fig. 4(e)-(i)]. In fact, the secondary flow, the main velocity and temperature distributions [Figs. 6(b)-(i),(ii),(iii)] resemble very much those in Figs. 4(e)-(i),(ii),(iii).

After the buoyancy-vortices disappear, the main flow still experiences a transition from that in Fig. 6(b)-(ii) to that in Fig. 6(c)-(ii) upon increasing the rotation speed further. This is similar to the transition from Fig. 4(e)-(ii) to Fig. 4(f)-(ii). It is noted that the secondary flow, the main flow and temperature distributions in Figs. 6(c)-(i),(ii),(iii) are qualitatively similar to those in Figs. 4(f)-(i),(ii),(iii). 

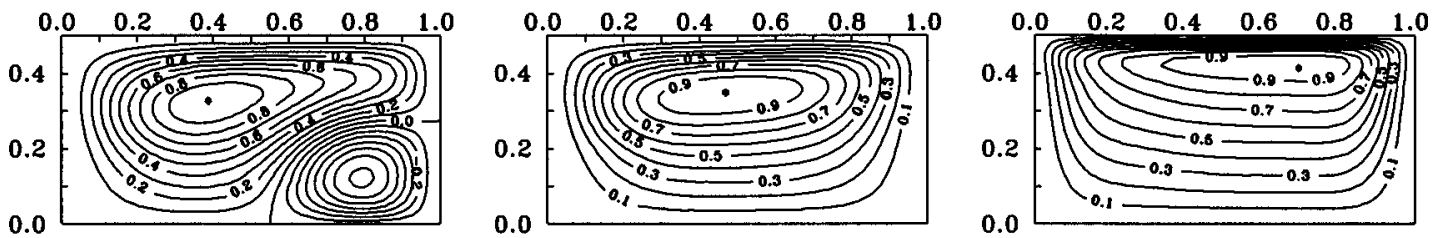

(i) Secondary flow patterns
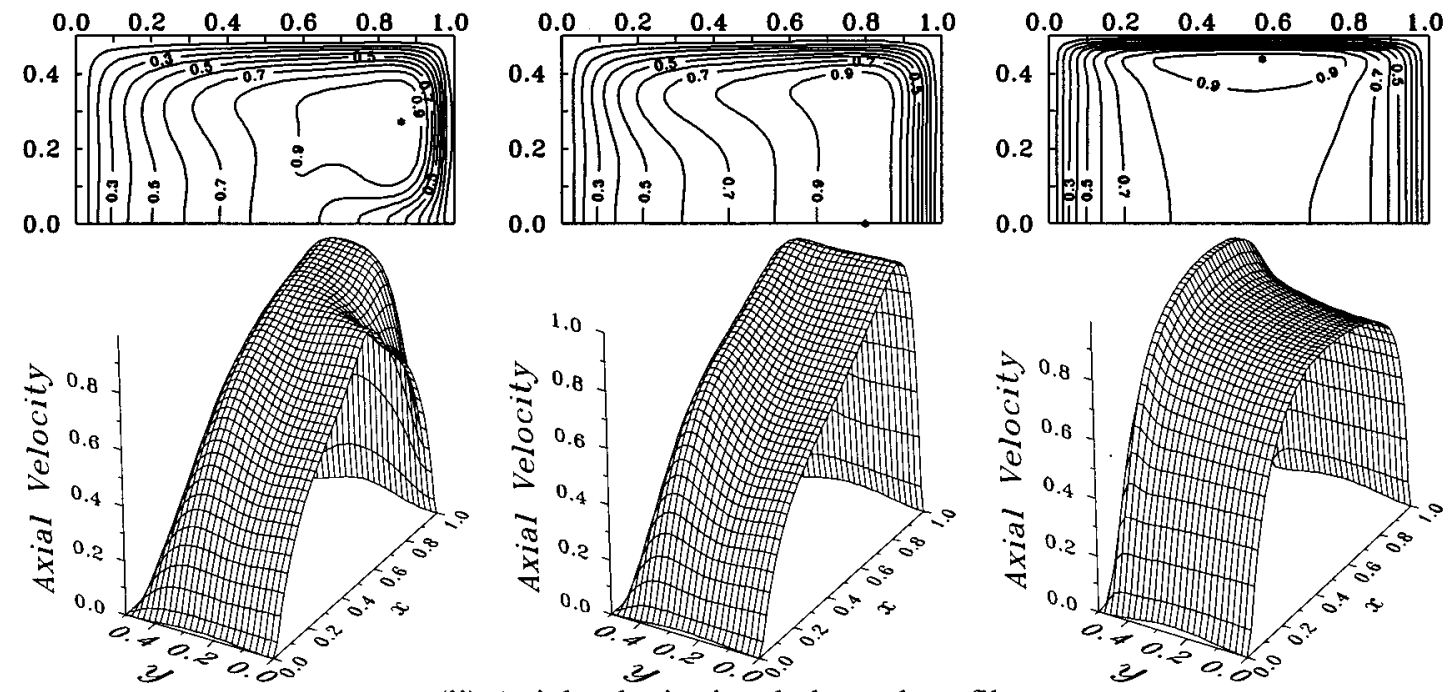

(ii) Axial velocity isopleths and profiles
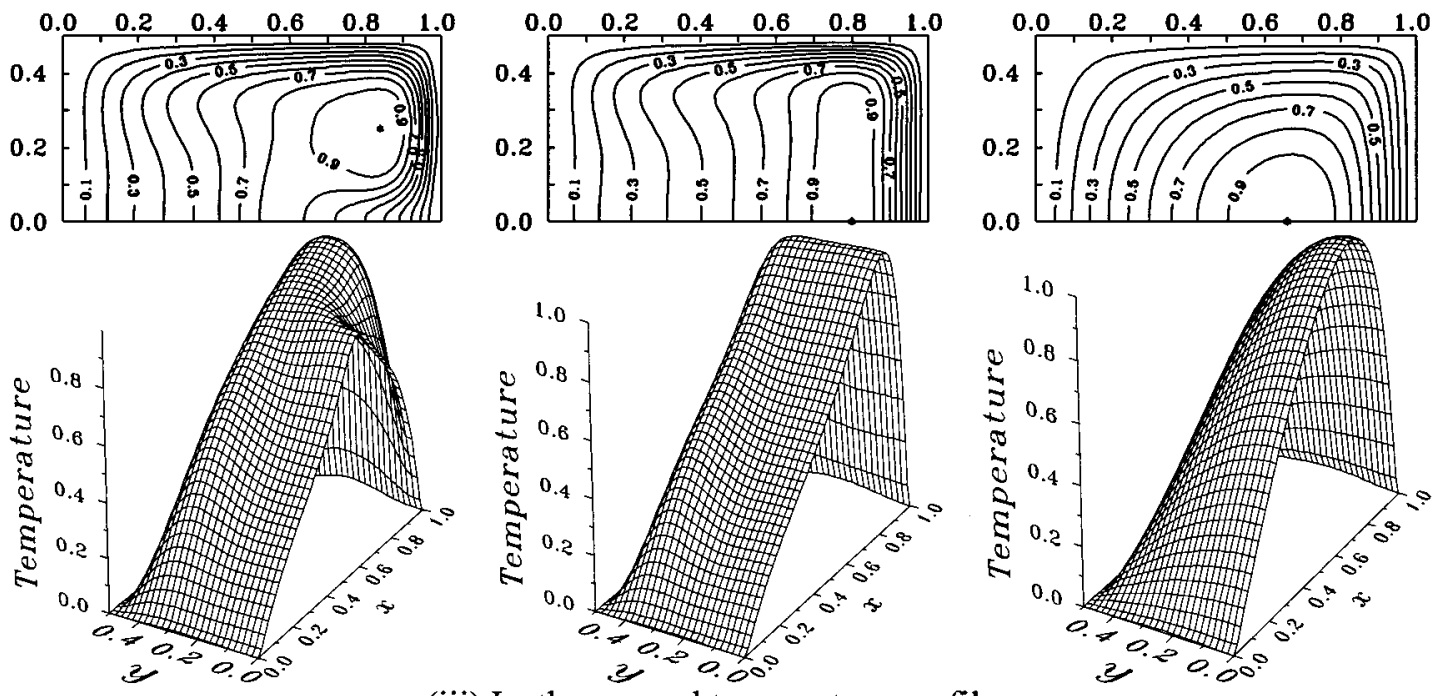

(iii) Isotherms and temperature profiles

(a) $\begin{aligned} L_{1}= & 0.1 ; \operatorname{Re}=379 ; R o=0.0176 \\ & (10.02 ; 0.0312 ; 16.75)\end{aligned}$ (b) $L_{1}=5 ; \operatorname{Re}=363 ; R o=0.918$

$(11.62 ; 0.0294 ; 16.88)$ (c) $L_{1}=70 ; \operatorname{Re}=181 ; R o=25.78$

$(3.749 ; 0.0131 ; 13.45)$

FIG. 6. Flow transitions and temperature distributions at $\gamma=1, \sigma=0.02, \operatorname{Pr}=0.7, D k=100$ and $L_{2}=5\left(R a_{\Omega}=8 \times 10^{3}\right)$. [Three values for each case are the maxima of the absolute value of the stream function $\left(|\psi|_{\max }\right)$, axial velocity $\left(w_{\max }\right)$ and temperature $\left(t_{\max }\right)$.]

\section{The cooling case with $L_{2}<0$}

If the fluid is cooled, the inward buoyancy force counteracts the centrifugal and Coriolis forces in the plane of the cross section. The flow situation is more complicated. The flows in various regions of the parameter can be different in nature. Figure 8 shows the secondary flow patterns, axial velocity isopleths and profiles, and isotherms and tempera- ture profiles for several representative values of $L_{1}$ at $\sigma=0.02, D k=100$ and $L_{2}=-5$. Shown in Fig. 8(a)-(i) is the stream function contour of the secondary flow, with an additional pair of vortices shown in the center part near the inner wall. This additional pair of vortices results from the buoyancy force instability which is similar to the centrifugal instability in the Dean problem or the Coriolis instability in the 

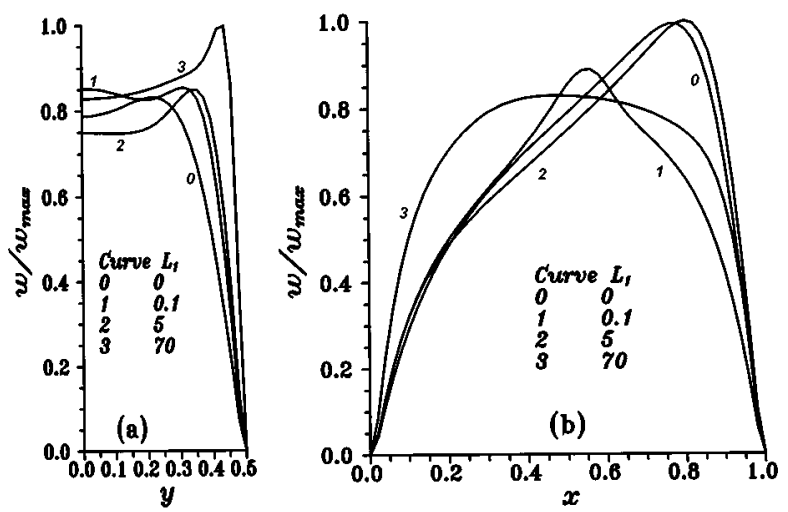

FIG. 7. Axial velocity distributions at $\gamma=1, \sigma=0.02, \operatorname{Pr}=0.7, D k=100$ and $L_{2}=5\left(R a_{\Omega}=8 \times 10^{3}\right)$ : (a) along the vertical centerline of the channel; (b) along the horizontal centerline of the channel.

Coriolis problem. They are called buoyancy-vortices in this paper. Note that the inward buoyancy forces cause the buoyancy-vortices to appear near the inner wall. This is different from the heating case, as shown in Fig. 6(a)-(i).

The presence of the buoyancy-vortices gives rise to a highly disturbed main flow field [Fig. 8(a)-(ii)] and temperature field [Fig. 8(a)-(iii)], with strong inflectional profiles developing in both $X$ and $Y$ directions [Fig. 8(a)-(ii) and curve 1 in Fig. 9]. This may result in a secondary instability, as discussed previously. The most striking feature of the buoyancy-vortices shown in Fig. 8(a)-(i) is that they appear in the low pressure side (inner wall) rather than the usual high pressure side (outer wall). This is indicated by the isopiestic contours which are not shown here. ${ }^{3}$ The secondary flow, axial velocity and temperature distributions [Fig. 8(a)] are qualitatively similar to those shown in Fig. 4(d) and Fig. 6(a) by interchanging the inner wall with the outer wall.

Upon increasing the value of $L_{1}$ while maintaining the same values for the other parameters, the buoyancy vortex pair shown in Fig. 8(a)-(i) disappears, and the secondary flow reduces to one-pair counter-rotating configuration [Fig. 8(b)-(i)] with circulating direction opposite to that shown in Figs. 4(a),(b). In fact, the buoyancy-vortices shown in Fig. 8(a)-(i) results from the break-up of this one-pair vortex flow due to buoyancy-force instability. By interchanging the inner and outer walls, the secondary flow, axial velocity and temperature distributions may be regarded to be qualitatively similar to those shown in Figs. 4(a),(b). The flow in the channel core is ageostrophic, i.e. pressure gradients are balanced by both convective inertial force and Coriolis force. The reverse direction of the secondary flow indicates that the secondary flow is still dominated by the buoyancy force.

If the Coriolis force is now increased further such that the resulting force of the centrifugal and Coriolis forces is of the same order of magnitude as the buoyancy force, the corner vortices occur and grow with circulating direction opposite to that of the vortices in the core region [Fig. 8(c)-(i)]. The flow in the channel core is neither ageostrophic nor geostrophic. The viscosity effect is not confined in a thin layer along the walls, and exists in the whole cross section of the channel. No counter-part exists in the cases with $L_{2} \geqslant 0$.
However, similar results were also found for the case of a circular cross section using the perturbation method. ${ }^{3,23}$ An important feature of the axial velocity in this flow region is the appearance of strong inflectional profiles [Fig. 8(c)-(ii) and curve 3 in Fig. 9]. This suggests a possible secondary instability problem. No such analysis appears to have been made in the past.

When two or more body forces are directed in the opposite direction and almost cancel each other, the principle of exchange of stabilities may not be valid, and one can anticipate that a time dependent instability sets in. This has been studied theoretically for high spanwise aspect ratio TaylorDean flow $^{50}$ and rotating curved channel flow, ${ }^{51}$ and experimentally for rotating curved channel flow at aspect ratios of 1 and 10.,46 The secondary flow was experimentally observed as multiple pairs of vortices which are very similar to that in Fig. 8(c)-(i), but are oscillating in time. Shown in Fig. 10 are additional secondary flow patterns from such flows for the other cases, including some cases with negative rotation.

When the rotation becomes more rapid, two corner vortices shown in Fig. 8(c)-(i) merge together and push the vortices in the center of the cross section to the outer wall. At the same time, two vortices near the center in Fig. 8(c)-(i) merge together and form one-pair of counter-rotating vortices near the center part of the outer wall. They are called merging-vortices in this paper (a more detailed study shows that the merging-vortices already form at $L_{1}=12 ; \mathrm{Wang}^{3}$ ). Although the secondary flow in Fig. 8(d)-(i) looks quite similar to that in Fig. 4(d) or Fig. 6(a), they are different in terms of the mechanism responsible for the appearance of an additional pair of vortices.

A break-up of the Ekman-vortices is associated with the Coriolis-vortices due to the Coriolis instability whereas the merging together of the vortices appearing in the region where the centrifugal, Coriolis and buoyancy forces just neutralize each other, characterizes the formation process of the merging-vortices. Here "just neutralizing" each other means that one of the three forces (termed as A-force, and it represents the buoyancy force for the cases shown in Fig. 8) cancels the other two forces (referring the resulting force of these two forces as the B-force), and the A-force and B-force have the same order of magnitude. The difference in the mechanism results in a smaller size of the merging-vortices than that of the Coriolis-vortices. Furthermore, the axial velocity and temperature distributions are also qualitatively different [see Figs. 4(d)-(ii),(iii), Fig. 6(a)-(ii),(iii), and Figs. 8(d)-(ii),(iii)].

It is interesting to note that the maximum of the velocity profile of curves 3 and 4 in Fig. 9(b) is positioned almost symmetric around the vertical centerline $x=0.5$. So we might expect that for some value of $L_{1}$ between 11.5 and 13.5 the profile is almost parabolic, with a maximum at $x=0.5$. At this value of $L_{1}$, the body forces are almost cancelling each other. A more detailed study in $\mathrm{Wang}^{3}$ shows that this would appear around $L_{1}=11.75$ (between 11.5 and 12).

Upon increasing the values of $L_{1}$ further, the mergingvortices disappear and the secondary flow becomes a slightly asymmetric one-pair vortex configuration [Fig. 8(e)-(i)]. The 

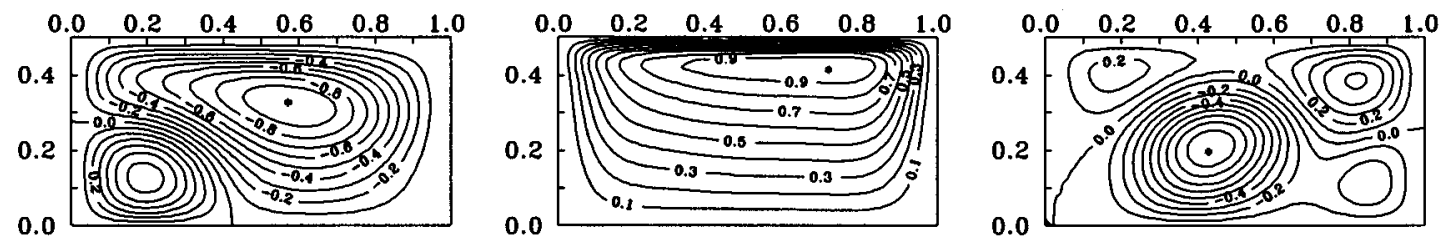

(i) Secondary flow patterns
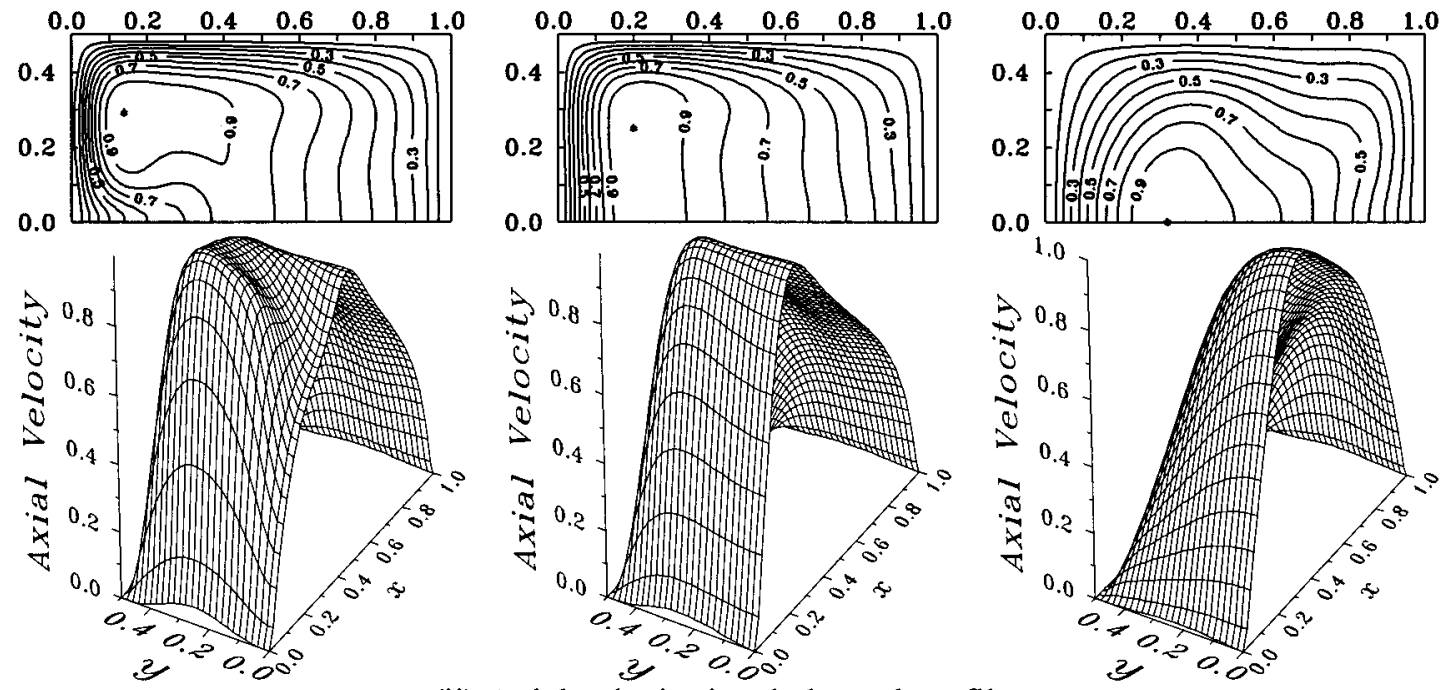

(ii) Axial velocity isopleths and profiles
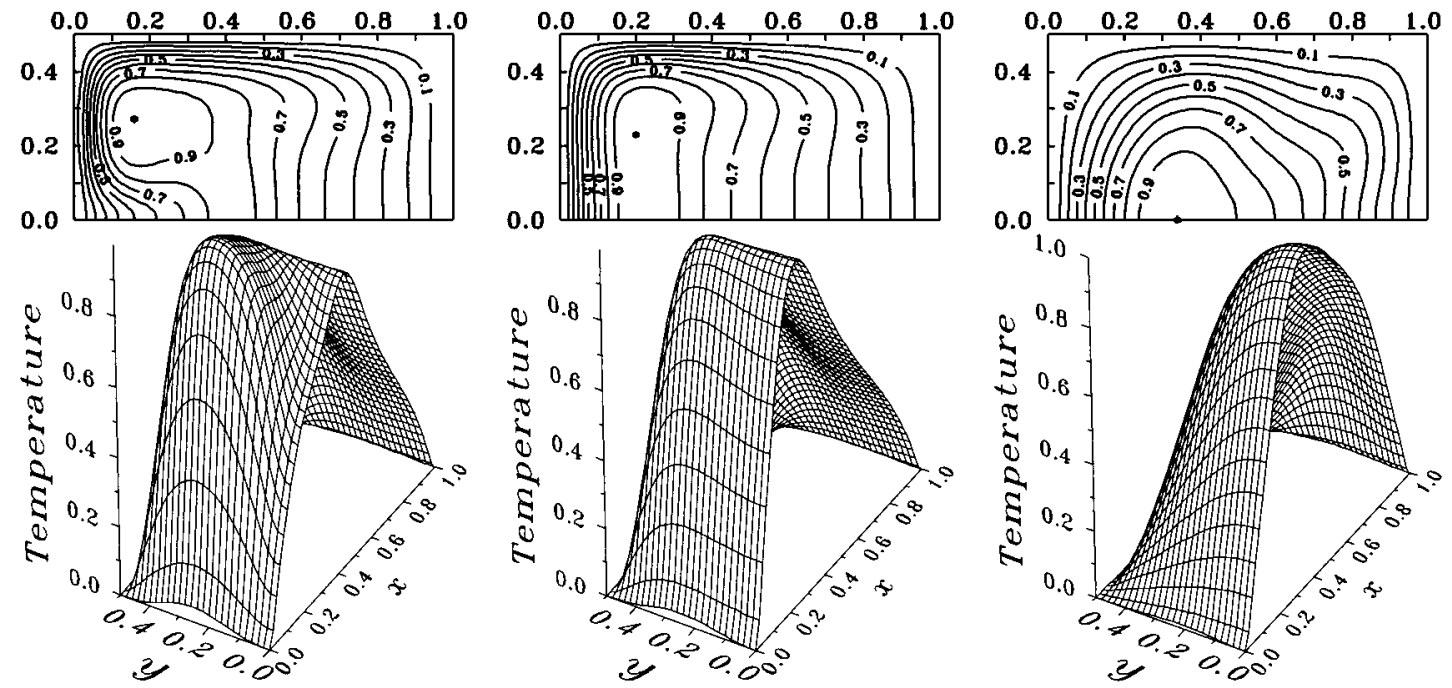

(iii) Isotherms and temperature profiles
(a) $L_{1}=1 ; \operatorname{Re}=401 ; R o=0.166$
(b) $L_{1}=3 ; \operatorname{Re}=457 ; R o=0.438$
$(9.003 ; 0.0341 ; 18.86)$
$(9.519 ; 0.0414 ; 25.21)$
(c) $L_{1}=11.5 ; \operatorname{Re}=652 ; R o=1.176$
$(2.716 ; 0.0688 ; 67.66)$

FIG. 8. Flow transitions and temperature distributions at $\gamma=1, \sigma=0.02, \operatorname{Pr}=0.7, D k=100$ and $L_{2}=-5\left(R a_{\Omega}=-8 \times 10^{3}\right)$. [Three values for each case are the maxima of the absolute value of the stream function $\left(|\psi|_{\max }\right)$, axial velocity $\left(w_{\max }\right)$ and temperature $\left(t_{\max }\right)$.]

axial velocity profile assumes a Taylor-Proudman configuration [Fig. 8(e)-(ii)] with a maximum located on the horizontal centerline. A similar profile is also observed for the temperature [Fig. 8(e)-(iii)].

Upon increasing the value of $L_{1}$ further, the secondary flow remains qualitatively unchanged [Fig. 8(f)-(i)]. How- ever, the dominant Coriolis force causes the main flow to have a bar-convex dumbbell-like profile with three high velocity regions [Fig. 8(f)-(ii)]. A geostrophic flow is observed in the channel core and Stewartson layers. The temperature profile [Fig. 8(f)-(iii)], however, remains qualitatively similar to that shown in Fig. 8(e)-(iii). 

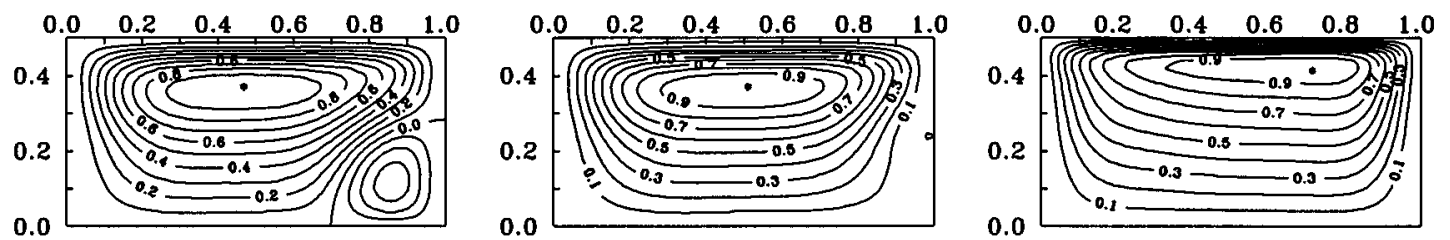

(i) Secondary flow patterns
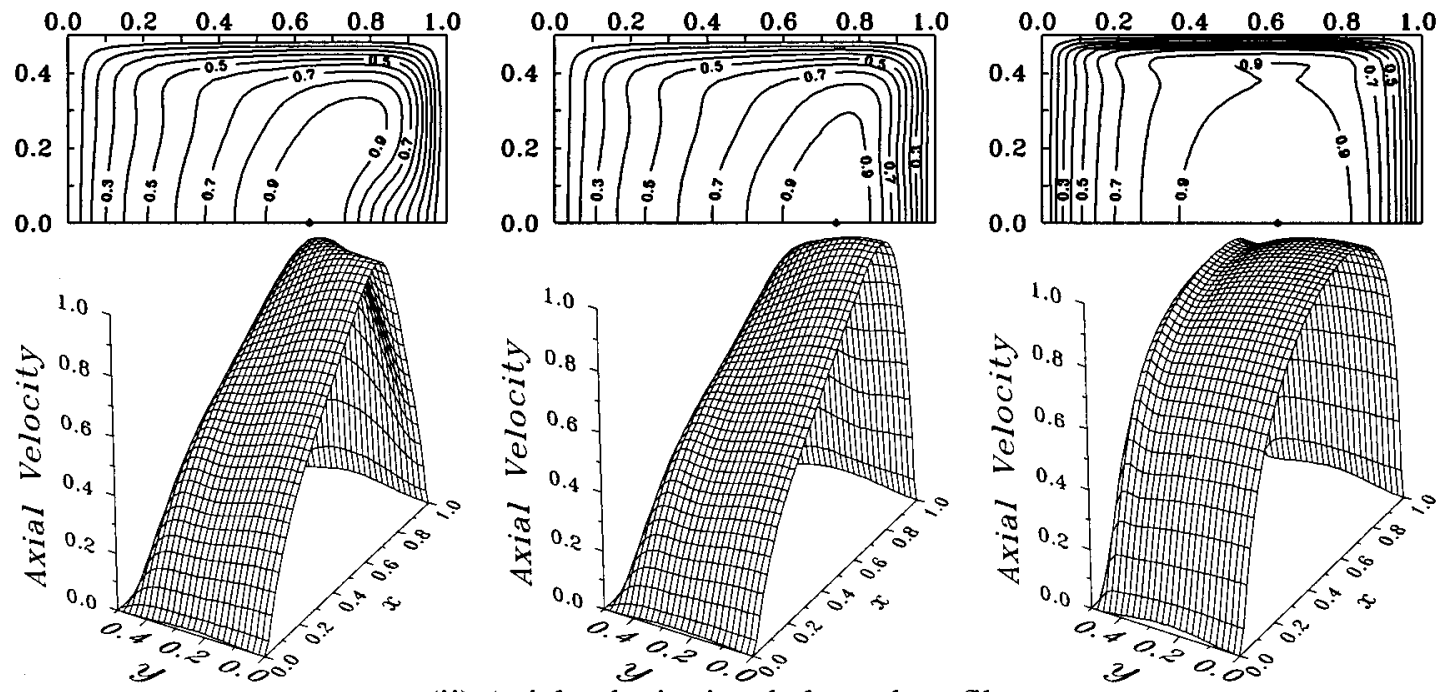

(ii) Axial velocity isopleths and profiles
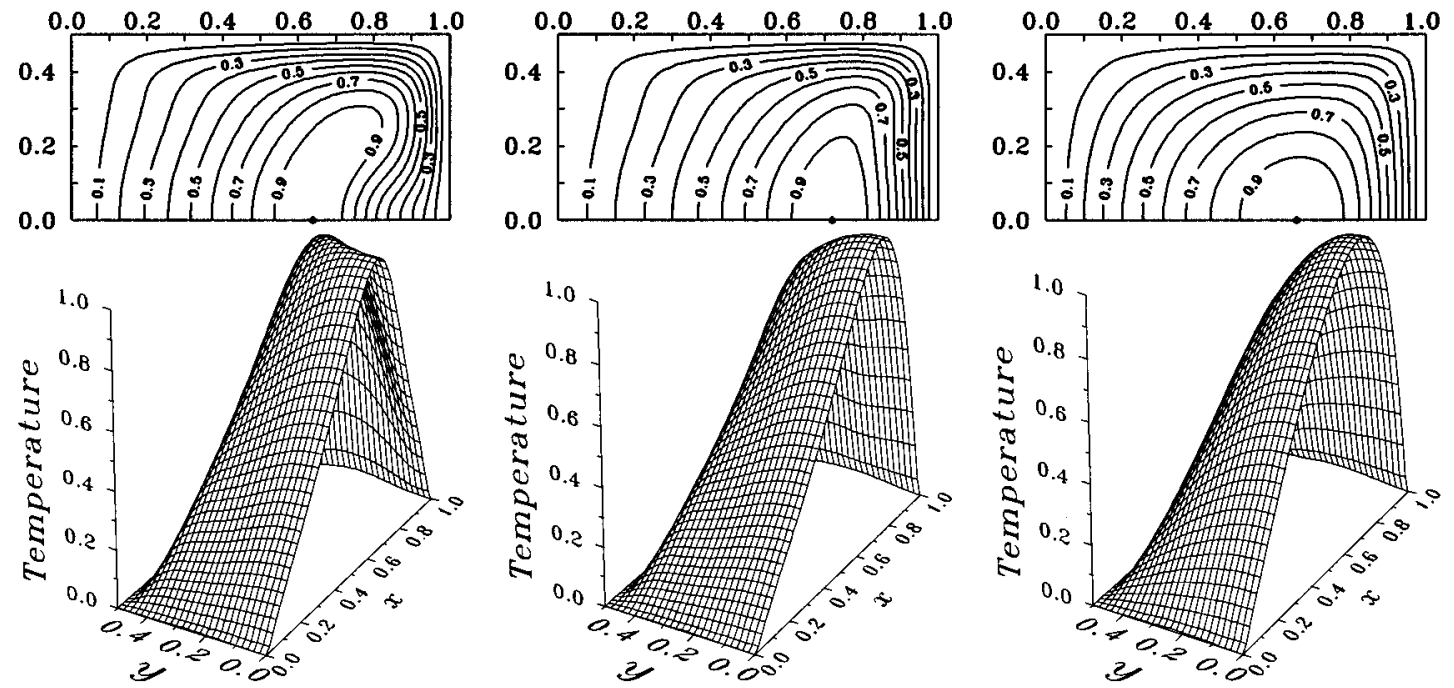

(iii) Isotherms and temperature profiles

(d) $L_{1}=13.5 ; \operatorname{Re}=415 ; R o=2.169$

$(5.053 ; 0.0367 ; 27.20)$ (e) $L_{1}=15 ; \operatorname{Re}=406 ; R o=2.463$

$(5.463 ; 0.0373 ; 29.37)$ (f) $L_{1}=70 ; \operatorname{Re}=213 ; R o=21.91$

$(3.430 ; 0.0148 ; 17.14)$

FIG. 8. (Continued.)

\section{B. The disappearance and reappearance of Dean-, Coriolis- and buoyancy-vortices}

The potential sources of instability for flow in rotating curved channels are centrifugal force, Coriolis force and buoyancy force. The instability from such body forces is in the form of streamwise-oriented vortices, i.e., Dean-vortices, Coriolis-vortices and buoyancy-vortices. The onset of these vortices receives much attention in recent years for the Dean,
Coriolis and mixed convection problems, respectively. Their disappearance and reappearance, however, have in general suffered comparative neglect. Very little information can be found in the published literature.

Dean-vortices, Coriolis-vortices and buoyancy-vortices perform differently in terms of their characteristics of the disappearance and reappearance although they share some similarities as observed by many investigators. Coriolis- 

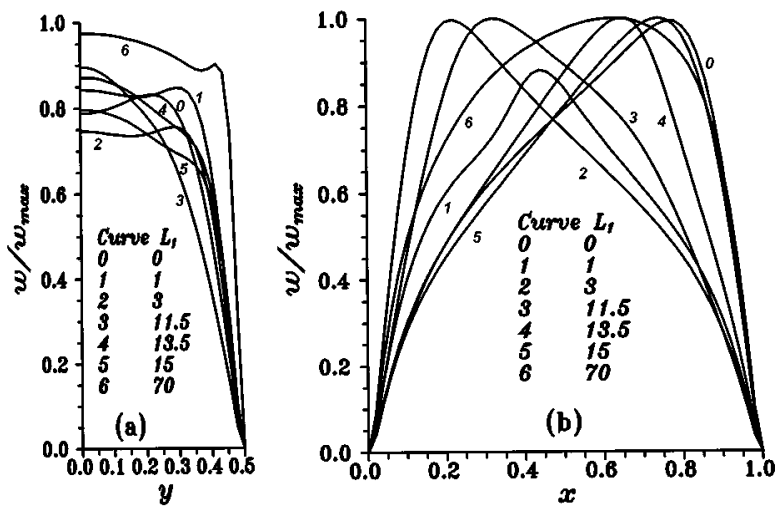

FIG. 9. Axial velocity distributions at $\gamma=1, \sigma=0.02, \operatorname{Pr}=0.7, D k=100$ and $L_{2}=-5\left(R a_{\Omega}=-8 \times 10^{3}\right)$ : (a) along the vertical centerline of the channel; (b) along the horizontal centerline of the channel.

vortices [Fig. 4(d)-(i)] disappear upon increasing the Coriolis force. The secondary flow restabilizes to a one-pair vortex flow [Fig. 4(e)-(i)], as discussed in the last section. If the Coriolis force is increased further, this one-pair vortex structure changes to another one-pair vortex flow [Fig. 4(f)-(i)]. However, no reappearance of the Coriolis-vortices is observed.

Figure 11 shows the disappearance and reappearance of the Dean-vortices [Fig. 11(i)] and buoyancy-vortices [Fig. 11(ii)]. For the stationary curved channel, one-pair vortex flow [Fig. 11(a)-(i)] becomes unstable with respect to the centrifugal instability upon increasing the centrifugal force sufficiently. The Dean-vortices are being set up in the region near the center of the outer wall [Fig. 11(b)-(i)]. Upon increasing the centrifugal force further up to that with $R e=5583$, no disappearance or reappearance is observed in the present calculation [Fig. 11(c)-(i)].

Figure 11(ii) shows the manner in which the buoyancy force affects the disappearance and reappearance of the buoyancy-vortices. Upon increasing the buoyancy-force, the buoyancy instability induces the buoyancy-vortices in the center part near the inner wall or outer wall [Fig. 11(e)-(ii) or Fig. 11(i)-(ii)] depending on whether the fluid is cooled or heated. The secondary flow changes from the original onepair vortex flow [Figs. 11(f),(g),(h)-(ii)] to two-pair vortex flow [Figs. 11(e),(i)-(ii)] [note that the circulation direction of the vortex in (f) is opposite to those in $(\mathrm{g}),(\mathrm{h})]$. Upon increasing the buoyancy force further, the buoyancy-vortices disappear. The secondary flow restabilizes to one-pair vortex configuration [Figs. 11(c),(d),(j)-(ii)]. When the buoyancy force is increased further, however, they reappear and remain in a large portion of the parameter space [Figs. 11(a),(b),(k),(l)-(ii)].

By the same argument as that in Sec. IV A 3 for flows in Figs. 8(c),(d)-(i), the streamwise-oriented counter-rotating vortex pair might disappear and the flow might be stable in a small region of $L_{2}$ in between $L_{2}=-2.5$ and $L_{2}=0$ [Figs. 11(f),(g)-(ii)] where the body forces are almost cancelling each other.

Some contradiction exists in the published literature about whether the Dean-vortices change size and shape with Dean number in fully developed flow region. Experiments by Bara et al. ${ }^{52}$ show that the size is about the same at all Dean numbers when the flows are fully developed. Such changes, however, are observed by Ligrani and Niver, ${ }^{41}$ Cheng et al. ${ }^{53}$ and Sugiyama et al. ${ }^{54}$ The present numerical calculations show that the Dean-vortices, Coriolis-vortices and buoyancyvortices change size and shape as the parameter changes, even in the fully developed flow region. This may be seen by comparing Figs. 11(b),(c)-(i) for the Dean-vortices, Figs. 4(c),(d)-(i) for the Coriolis-vortices and Figs. 11(a),(b),(e),(i),(k),(l)-(ii) for the buoyancy-vortices.

\section{The distributions of friction factor and Nusselt number}

For engineering applications, the most important results are the friction factor and the Nusselt number. Since the main flow and temperature fields determine the friction factor and Nusselt number, the flow transitions discussed in the last section will strongly affect the distributions of the friction factor and Nusselt number.

Following the usual definitions, the expression for the product of the friction factor and Reynolds number $f R e$ and Nusselt number $\mathrm{Nu}$ can be written based on the local axial velocity gradient or the temperature gradient at the wall as

$$
\begin{aligned}
& f R e=\frac{2}{w_{m}}\left(\frac{\partial w}{\partial n}\right)_{\text {wall }}, \\
& N u=\frac{1}{t_{b}}\left(\frac{\partial t}{\partial n}\right)_{\text {wall }},
\end{aligned}
$$

where $w_{m}$ and $t_{b}$ are mean axial velocity and bulk mean temperature, respectively.

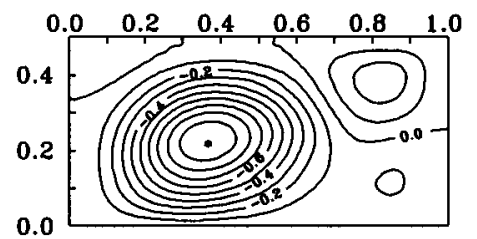

(a)

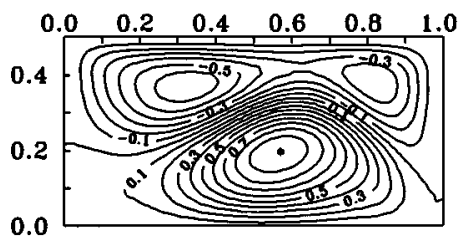

(b)

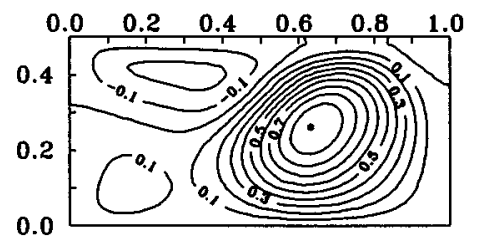

(c)

FIG. 10. Secondary flows when body forces just neutralize each other at $\gamma=1, \sigma=0.02$ and $\operatorname{Pr}=0.7$ : (a) $D k=100 ; L_{1}=10 ; L_{2}=-5 ; \operatorname{Re}=606$; $R o=1.100 ; R a_{\Omega}=-8 \times 10^{3} ;|\psi|_{\max }=4.416 ;$ (b) $D k=500 ; L_{1}=-0.7 ; L_{2}=0 ; R e=2612 ; R o=-0.0893 ; R a_{\Omega}=0 ; \quad|\psi|_{\max }=8.846 ; \quad(\mathrm{c}) \quad D k=500 ;$ $L_{1}=-2.0 ; L_{2}=1 ; R e=2147 ; R o=-0.311 ; R a_{\Omega}=1.6 \times 10^{4} ;|\psi|_{\text {max }}=14.55$. 


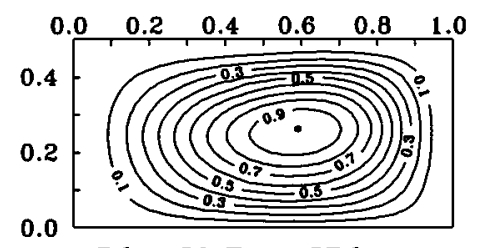

(a) $D k=50 ; \operatorname{Re}=576$;

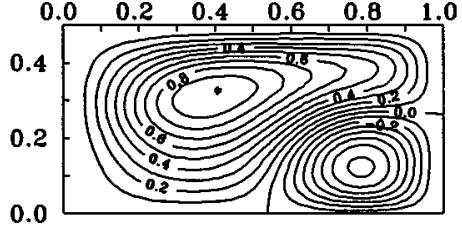

(b) $\begin{aligned} & D k=200 ; \operatorname{Re} \\ & |\psi|_{\max }=8.227\end{aligned}$

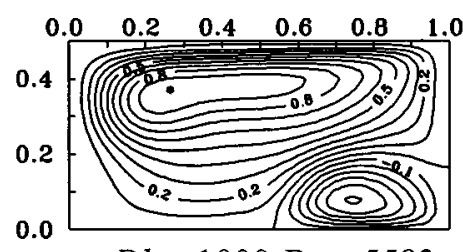

(c)

$|\psi|_{\max }=19.422$

(i) Dean-vortices $\left(\gamma=1, \sigma=0.01, \operatorname{Pr}=0.7, L_{1}=L_{2}=0\right)$

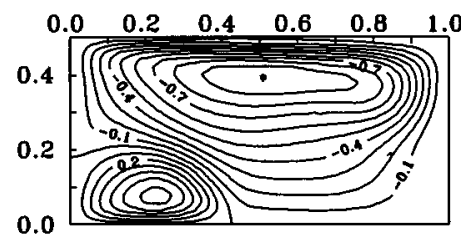

(a) $\begin{aligned} & L_{2}=-140 ; \operatorname{Re}= \\ & |\psi|_{\max }=21.561\end{aligned}$

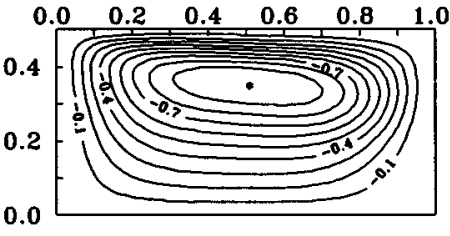

(d) $L_{2}=-15 ; \operatorname{Re}=368$; $|\psi|_{\max }=13.426$

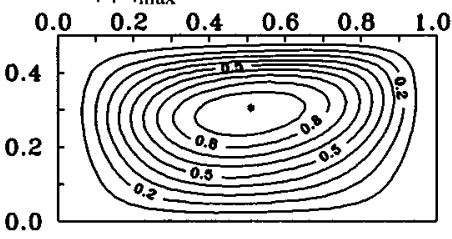

$L_{2}=0 ; \operatorname{Re}=494$

(g)

$|\psi|_{\max }=6.865$

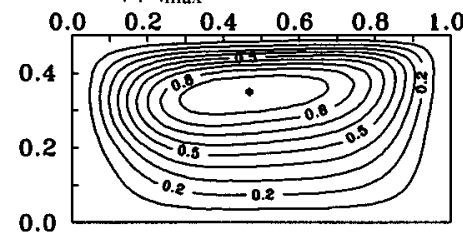

(j) $\begin{aligned} & L_{2}=10 ; \operatorname{Re}=367 ; \\ & |\psi|_{\max }=12.952\end{aligned}$

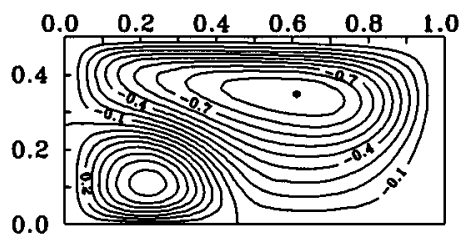

(b) $L_{2}=-17.5 ; \operatorname{Re}=334$ $|\psi|_{\max }=13.497$
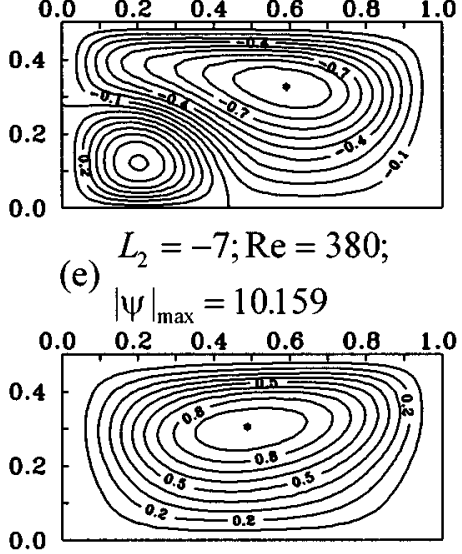

(h) $L_{2}=0.5 ; \operatorname{Re}=470$ $|\psi|_{\max }=7.842$

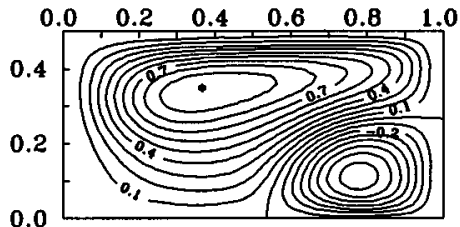

(k) $\begin{aligned} & L_{2}=12.5 ; \operatorname{Re}=335 \\ & \end{aligned}$

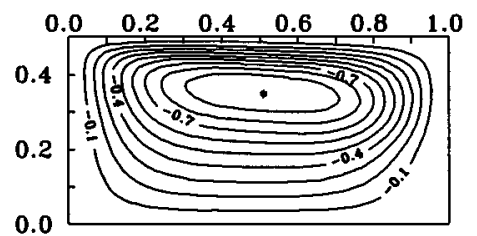

(c)

$L_{2}=-17.25 ; \operatorname{Re}=361$

$|\psi|_{\max }=13.811$

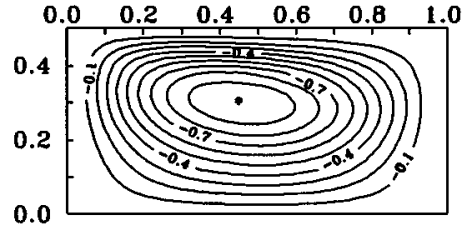

(f) $L_{2}=-2.5 ; \mathrm{Re}=477$;

$|\psi|_{\max }=8.369$

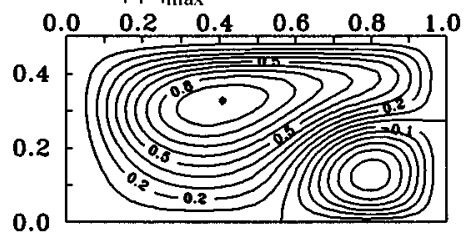

(i)

$$
L_{2}=2.5 ; \mathrm{Re}=400
$$

$|\psi|_{\max }=8.712$

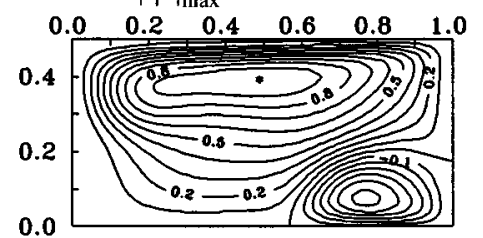

(1)

$$
L_{2}=140 ; \operatorname{Re}=247 ;
$$

$|\psi|_{\max }=22.197$

(ii) Buoyancy-vortices $\left(\gamma=1, \sigma=0.02, \operatorname{Pr}=0.7, D k=100, L_{1}=1\right)$

FIG. 11. Disappearance and reappearance of Dean-vortices and buoyancy-vortices.

The mean friction factor and Nusselt number can be obtained either by peripherally averaging the local values or by making the overall force and energy balance along the axis of the channel. The calculated values by these two methods were found to be in good agreement. The result presented in this paper is the average of the values obtained by these two methods.

The distributions of the friction factor $f R e$ and Nusselt number $N u$ along the upper half of the inner wall, upper wall and the upper half of the outer wall, are illustrated in Figs. 12(a), (b) and (c), respectively. They are shown on the basis of the values for a stationary straight channel [ $(f R e)_{0}=14.23$ and $\left.N u_{0}=3.608\right]$ to facilitate the understanding of the variations. The distributions are plotted for six values of $L_{1}$ ranging from 1 (curve 1) to 70 (curve 6) at $\sigma=0.02, D k=100$ and $L_{2}=-5$. The corresponding flow patterns are illustrated in Fig. 8. Also shown in the figure are the friction factor and Nusselt number for a curved channel without rotation, i.e., $L_{1}=L_{2}=0$, denoted by the curve 0 .

The Dean-vortices, Coriolis-vortices and buoyancyvortices are of importance in the fundamental research of roll-cell instabilities. They also change the flow resistance 

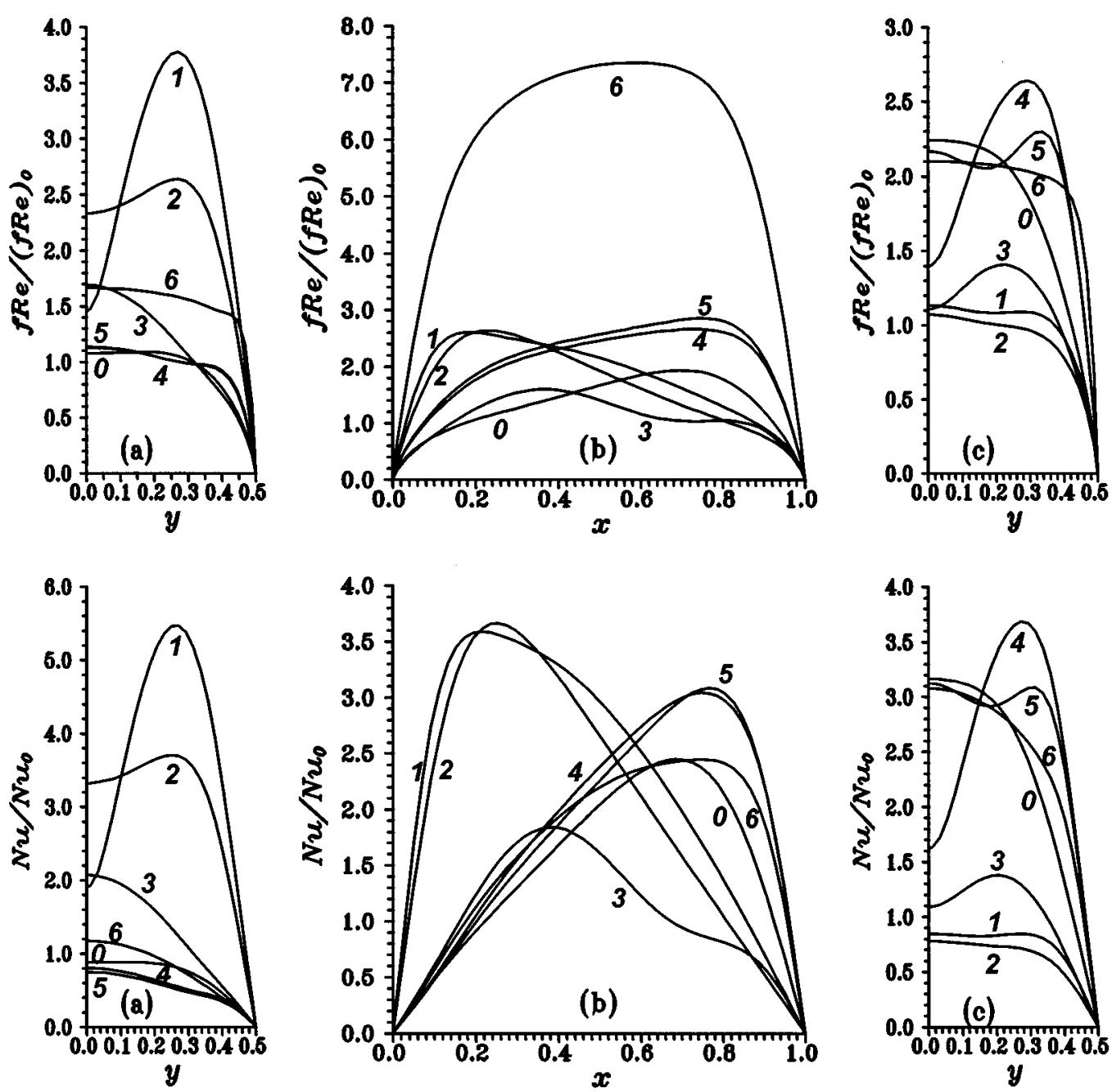

FIG. 12. Distributions of friction factor and Nusselt number at $\gamma=1, \sigma=0.02, \operatorname{Pr}=0.7, D k=100$ and $L_{2}=-5\left(R a_{\Omega}=-8 \times 10^{3}\right)$. (Curves $1-6$ correspond to $L_{1}=1,3,11.5,13.5,15$ and 70 ; curve 0 is for the case of $L_{1}=L_{2}=0$.) (a) Along the inner wall; (b) along the upper wall; (c) along the outer wall.

and heat transfer characteristics significantly. Curve 1 shows the friction factor and Nusselt number ratios with the buoyancy-vortices in secondary flow [Fig. 8(a)-(i)]. The similarity between the main flow and temperature distributions [Figs. 8(a)-(ii),(iii)] results in a similarity between the friction factor and the Nusselt number distributions. The asymmetry of the buoyancy-vortices, with respect to the vertical centerline, leads to different distributions of friction factor and Nusselt number at the inner wall from those at the outer wall.

The large velocity (temperature) gradient between high velocity (temperature) core and the inner wall [Figs. 8(a)(ii),(iii)] results in a peak of the local friction factor (Nusselt number) in that region along the inner wall. A low friction factor (Nusselt number) region is observed in the center of the inner wall. This is induced by the outerwash isovels (isotherms) in that region. The quite uniform axial velocity (temperature) along the outer wall leads to a nearly constant local friction factor (Nusselt number) over a wide region of the outer wall. The friction factor (Nusselt number) along the upper wall reaches a peak near the inner wall because a large velocity (temperature) gradient exists between high velocity (temperature) core and the upper wall as shown in Figs. 8(a)(ii),(iii).

Curve 2 illustrates the friction factor and Nusselt number ratio corresponding to one-pair vortex flow in Fig. 8(b). The distribution along the outer wall is qualitatively similar to that with the buoyancy-vortices (curve 1) but with lower values in general. The similarity also holds for those along the upper wall with peaks shifted toward the outer wall. The distribution of the friction factor (Nusselt number) along the inner wall, however, experiences a dramatic change in response to the disappearance of the buoyancy-vortices. The peak region in curve 1 is flattened by increasing the values in the region near the center of the inner wall and decreasing those in the other region (curve 2). Curve 3 shows the friction factor and Nusselt number distribution in the flow region where the centrifugal, Coriolis and buoyancy forces just neutralize each other [Fig. 8(c)]. The friction factor (Nusselt number), along the inner wall, decreases monotonously from the center to the upper wall [Fig. 12(a)]. The corner vortex in the upper-outer corner increases the local friction factor (Nusselt number) along the upper wall in the portion near the outer wall, resulting in a local peak in that region [Fig. 


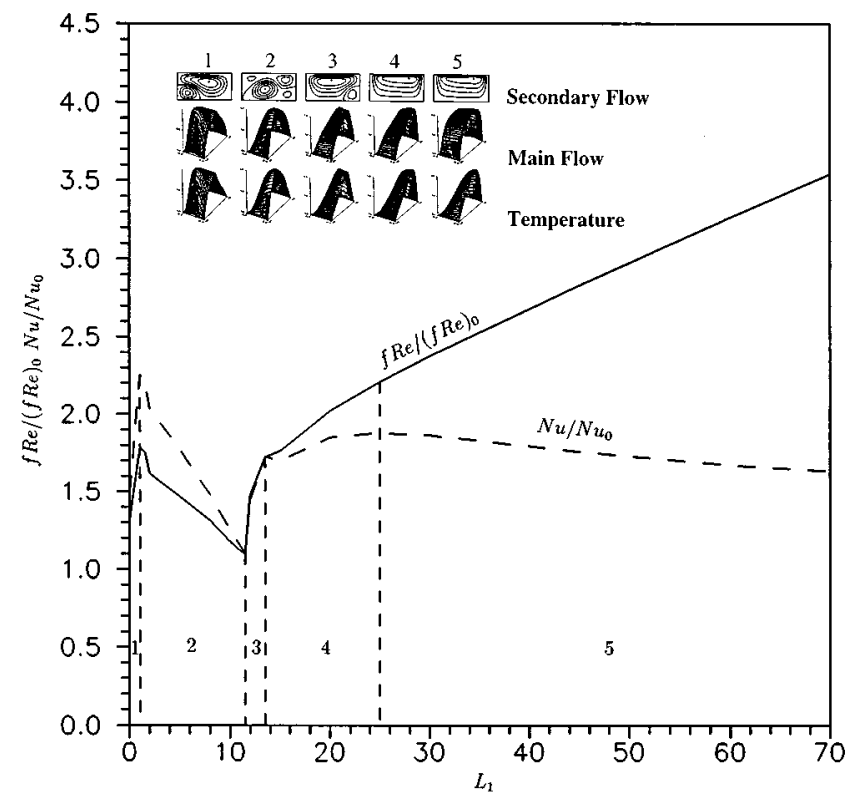

FIG. 13. Mean friction factor and Nusselt number at $\gamma=1, \sigma=0.02$, $\operatorname{Pr}=0.7, D k=100$ and $L_{2}=-5\left(R a_{\Omega}=-8 \times 10^{3}\right)$.

12(b)]. And a decrease in peak value is observed in curve 3 [Fig. 12(b)]. Near the boundary between the upper-outer vortex and that located at the center part of the outer wall, the current impinges on the outer wall so that the local friction factor (Nusselt number), along the outer wall, is increased in that region. This results in a peak near the vortex boundary [curve 3 in Fig. 12(c)].

Once merging-vortices appear near the center part of the outer wall, the isovels (isotherms) along the inner wall become more sparsely spaced [Figs. 8(d)-(ii),(iii)]. Consequently, a dramatic drop in the friction factor (Nusselt number) occurs for curve 4 [Fig. 12(a)]. The more tightly spaced isovels (isotherms) along the upper wall, however, result in a high local friction factor (Nusselt number) along the upper wall, as shown by curve 4 in Fig. 12(b). The most significant effect of the merging-vortices on the friction factor and Nusselt number occurs along the outer wall, as shown by curve 4 in Fig. 12(c). The striking feature is that the peaks for both friction factor and Nusselt number are located at the boundary between Ekman-type-vortices and merging-vortices. This results from a current impinging on the outer wall in that region [see Fig. 8(d)-(i)].

After the merging-vortices disappear upon increasing the value of $L_{1}$, friction factor and Nusselt number along the inner and upper walls are qualitatively similar to those with the presence of the merging-vortices. And they are quantitatively increased as compared with those with the presence of the merging-vortices. This may be seen by comparing curves 5 and 6 with curve 4 in Figs. 12(a) and (b). The distributions of the friction factor and Nusselt number along the outer wall, however, experience a qualitative change due to the disappearance of the merging-vortices, as shown by curves 5 and 6 in Fig. 12(c). It is observed that the peak region in curve 4 is flattened through increasing local friction factor and Nusselt number in the center part of the outer wall.

Figure 13 shows the result for the mean friction factor and Nusselt number represented by the solid and dotted lines, respectively. They are plotted in the form of $f R e /(f R e)_{0}$ and $N u / N u_{0}$ against $L_{1}$ at $\sigma=0.02$, $\operatorname{Pr}=0.7, D k=100$ and $L_{2}=-5$. The characteristic flow regimes for the secondary flow, the main flow and the temperature are also shown for reference. It is noted that the flow transitions significantly affect the mean friction factor and Nusselt number. The appearance of the buoyancy-vortices substantially increases the friction factor and Nusselt number. And the increase in $N u$ is more appreciable.

When the flow is in the region where centrifugal, Coriolis and buoyancy forces just neutralize each other, both friction factor and Nusselt number approach those values for forced convection in a stationary straight channel. This is because the secondary flow becomes weaker due to the impaired interaction among the forces. The friction factor and Nusselt number, however, increase significantly once again once the flow moves to the region with merging-vortices. An interesting feature about the friction factor and Nusselt number in this flow region is that their ratios, with respect to those for the stationary straight channel, are nearly identical. After the disappearance of the merging-vortices, the friction factor ratio increases proportionally with $L_{1}$ as shown in Fig. 13. The Nusselt number ratio, however, increases at first, but then decreases with $L_{1}$ slightly. The different profiles of the axial velocity and temperature contribute to the different variations of the friction factor and Nusselt number in this Coriolis force dominated flow region.

It is well to compare the friction factor ratio and Nusselt number ratio in Fig. 13 from a point of view of practical engineering. The Nusselt number ratio is higher than that of the friction factor for the flows shown in Figs. 8(a)-(c). They are nearly identical if the flow is in the region with the merging-vortices. When the flow is in the region as shown in Figs. 8(e),(f), however, the Nusselt number ratio is much lower than the friction factor ratio.

\section{CONCLUDING REMARKS}

Flow transition phenomena and combined free and forced laminar convective heat transfer were studied numerically for fully developed flow in the square channels with both curvature and rotation, using the finite-volume method. Curvature and rotation, in conjunction with heating or cooling, introduce the centrifugal force, Coriolis force and buoyancy force in the momentum equations, which describe the relative motion of fluids with respect to the channel. Such body forces cause similar instabilities (centrifugal instability, Coriolis instability and buoyancy instability) in forms of streamwise oriented vortices. In addition, these forces may either enhance or impede each other in the cross-plane depending on the directions of the rotation and heat flux. This produces a rich transition structure for both secondary flow and pressure-driven main flow. The present investigation is confined to examine this structure in the hydrodynamically and thermally fully developed laminar flow region. The work is also limited to the symmetric flow with respect to the horizontal centerline of the cross section by imposing a sym- 
metric condition on that line. The results presented in this paper are for the case of the square cross section of the channel with positive rotation only.

Despite the assumptions made in the present investigation, the calculations cover a rather wide range of the parameters. In particular, the Reynolds number reached up to about six thousands. The rotation rates approached previously studied asymptotic limits of weak rotation and strong rotation where viscous force or Coriolis force dominates. Several flow patterns, hitherto unknown, are revealed in the present study. A one-pair vortex flow with an ageostrophic, virtually inviscid core occurs between a viscous force dominated onepair vortex flow and two-pair vortex flow with the presence of the Dean-vortices, Coriolis-vortices or buoyancy-vortices. Another two kinds of one-pair vortex flows exist after the disappearance of the Coriolis-vortices upon increasing the Coriolis forces further. The axial velocity profile for the first one assumes a Taylor-Proudman configuration in the core region, with one maximum located on the horizontal centerline. That for the second one is dumbbell-like with two maxima or bar-convex dumbbell-like with three high velocity regions. The flow in the core region is also geostrophic for the second kind of one-pair vortex flow.

When the fluid is cooled, there exists a parametric region where, overall, the effect of the inward buoyancy force just neutralizes those of the outward centrifugal and Coriolis forces. In this region, new vortices appear and grow around the corners, squeezing the circulation due to the centrifugal and Coriolis forces to the central portion of the cross section of the channel. The flow thus exhibits a two-pair, three-pair or four-pair structure. Here, the two-pair vortex structure is qualitatively different from the two-pair vortex families encountered in the Dean problem or Coriolis problem.

The remaining domain of the two-pair vortex families may still be divided into two different regions. The distinction is based on the mechanisms responsible for the appearance of an additional pair of vortices. A break-up of the Ekman-vortices (due to the centrifugal, Coriolis or buoyancy force instabilities) is associated with one case, whereas in the other case the merging together represents the forming process of the vortices appearing in the region where the centrifugal, Coriolis and buoyancy forces just neutralize each other. The additional pair of vortices formed in the second mechanism is called merging-vortices in this paper. They appear in the region near the center of the outer wall (high pressure side) in the case with positive rotation. The circulation direction is the same as that of the Dean-vortices. The size, however, is smaller than that of the Dean-vortices.

The vortices formed in the first mechanism include the Dean-vortices, Coriolis-vortices and buoyancy-vortices. Their shape and size change with the dynamical parameters even in the fully developed flow region. In addition, the Dean-vortices, Coriolis-vortices and buoyancy-vortices behave differently in some aspects although they share some similarities, as noted by many investigators. The disappearance of the Coriolis-vortices or buoyancy-vortices is observed with increasing Coriolis or buoyancy force. No such phenomenon is observed for the Dean-vortices. Furthermore, the reappearance of the buoyancy-vortices, upon increasing the buoyancy force further, is also found in this study. Another important difference is the locations of the vortices. Although the Dean-vortices and Coriolis-vortices always appear on the high pressure side (outer wall) in the case of the positive rotation, the buoyancy-vortices may appear in the high pressure side (outer wall) or low pressure side (inner wall) depending on whether the fluid is heated or cooled. If the fluid is heated, they show up near the center of the outer wall with the same direction of circulation as those of the Dean-vortices and Coriolis-vortices. When the fluid is cooled, however, the inward buoyancy forces cause them to appear near the center of the inner wall with an opposite direction of circulation. The most striking feature is that the fluid near the inner wall still remains low pressure, even with the existence of the buoyancy-vortices.

When the fluid is cooled, the inward buoyancy forces cause the direction of the secondary flow to reverse by overcoming the outward centrifugal and Coriolis forces in the plane of the cross section. The flow reversal occurs by passing through a multi-pair vortex flow region where overall the effect of the buoyancy force just neutralizes those of the centrifugal and Coriolis forces.

The friction factors and Nusselt numbers are significantly affected by the flow transitions. In particular, the Dean-vortices, the Coriolis-vortices, the buoyancy-vortices and the merging-vortices substantially change the distributions of the local friction factor and Nusselt number with a remarkable increase in their mean values.

The new vortex flows, revealed in the present study, suggest possible further research concerning their instability since usually an inflectional profile of the main flow is associated with them. Such a study is considered to be complex because of the full three-dimensional form of the resulting disturbance equations, and is beyond the scope of the present study.

\section{ACKNOWLEDGMENTS}

Financial support from the Natural Sciences and Engineering Research Council of Canada through the operating grant programme is gratefully acknowledged. The authors are also indebted to the reviewers for their critical review on the original manuscript.

${ }^{1}$ W. D. Morris, Heat Transfer and Fluid Flow in Rotating Coolant Channels (Research Studies Press, Wiley, Chichester, 1981), pp. 27-31.

${ }^{2}$ W. R. Dean, "The stream-line motion of a fluid in a curved pipe," Philos. Mag. 5, 673 (1928).

${ }^{3} \mathrm{~L}$. Wang, "Fluid flow and heat transfer in rotating curved channels, Ph.D. thesis, Department of Mechanical Engineering, University of Alberta, 1995.

${ }^{4}$ In terms of the force ratios, $D_{m}=($ inertial force $) \times($ buoyancy force $) /$ $(\text { viscous force })^{2} ; D_{\Omega}=($ inertial force $) \times($ Coriolis force $) /(\text { viscous force })^{2}$. They play a similar role as that of the Dean number in the Dean problem for the mixed-convection problem and the Coriolis problem, respectively. Here we use $D$ to denote this analogous role, and subscripts $m$ and $\Omega$ to denote mixed-convection problem and Coriolis problem (effect of rotation $\Omega$ ).

${ }^{5} \mathrm{~K}$. H. Winters, “A bifurcation study of laminar flow in a curved tube of rectangular cross-section," J. Fluid Mech. 180, 343 (1987).

${ }^{6}$ K. Nandakumar, H. Raszillier, and F. Durst, "Flow through rotating rectangular ducts," Phys. Fluids A 3, 770 (1991).

${ }^{7}$ K. Nandakumar and H. J. Weinitschke, "A bifurcation study of mixed- 
convection heat transfer in horizontal ducts,' J. Fluid Mech. 231, 157 (1991).

${ }^{8}$ S. R. Sankar, K. Nandakumar, and J. H. Masliyah, "Oscillatory flows in coiled square ducts,' Phys. Fluids 31, 1348 (1988).

${ }^{9}$ K. Nandakumar and J. H. Masliyah, "Swirling flow and heat transfer in coiled and twisted pipes,' Adv. Transport Proc. 4, 49 (1986).

${ }^{10} \mathrm{G}$. J. Hwang and C. H. Chao, "Forced laminar convection in a curved isothermal square duct,' J. Heat Transfer 113, 48 (1991).

${ }^{11}$ W. J. Yang, S. Fan, and J. H. Kim, "Heat and fluid flow inside rotating channels,'” Appl. Mech. Rev. 47, 367 (1994).

${ }^{12}$ L. M. Hocking, "Boundary and shear layers in a curved rotating pipe,' J. Math. Phys. Sci. 1, 123 (1967).

${ }^{13}$ H. Ludwieg, "Die ausgebildete kanalströmung in einem rotierenden system,’ Ing. Arch. Bd. 19, 296 (1951).

${ }^{14} \mathrm{H}$. Ito and T. Motai, "Secondary flow in a rotating curved pipe,', Rep. Inst. High Speed Mech. 29, 33 (1974).

${ }^{15}$ H. Miyazaki, "Combined free and forced convective heat transfer and fluid flow in a rotating curved circular tube,' Int. J. Heat Mass Transfer 14, 1295 (1971).

${ }^{16} \mathrm{H}$. Miyazaki, "Combined free and forced convective heat transfer and fluid flow in a rotating curved rectangular tubes,' J. Heat Transfer 95, 64 (1973).

${ }^{17}$ S. Thangam and C. G. Speziale, "Numerical study of thermal convection in rotating channel flow,' Int. J. Num. Methods Fluids 5, 133 (1985).

${ }^{18}$ O. J. E. Matsson and P. H. Alfredsson, "The effect of spanwise system rotation on Dean vortices,' J. Fluid Mech. 274, 243 (1994).

${ }^{19}$ R. K. Shah and A. L. London, Laminar Flow Forced Convection in Ducts (Academic, New York, 1978).

${ }^{20}$ S. W. Hong, S. M. Morcos, and A. E. Bergles, "Analytical and experimental results for combined forced and free convection in horizontal tubes,' in Proceedings of the Fifth International Heat Transfer Conference, Vol. 3, p. 154 (1974).

${ }^{21}$ E. R. G. Eckert and R. M. Drake, Analysis of Heat and Mass Transfer (McGraw-Hill, New York, 1972), p. 525.

${ }^{22}$ In terms of the force ratios, $L_{1}$ and $L_{2}$ are the ratios of the Coriolis force and the buoyancy force over the centrifugal force, respectively. In particular, $L_{1}$ is proportional to $R_{c} \Omega / W_{1}$ which may be regarded as a modified rotation number.

${ }^{23} \mathrm{~L}$. Wang and K. C. Cheng, "Flow transitions and combined free and forced convective heat transfer in a rotating curved circular tube,' Int. J. Heat Mass Transfer (in press).

${ }^{24}$ S. V. Patankar, Numerical Heat Transfer and Fluid Flow (Hemisphere, New York, 1980).

${ }^{25}$ J. A. Baylis, "Experiments on laminar flow in curved channels of square section,' J. Fluid Mech. 48, 417 (1971).

${ }^{26}$ K. C. Cheng and M. Akiyama, "Laminar forced convection heat transfer in curved rectangular channels,', Int. J. Heat Mass Transfer 13, 471 (1970).

${ }^{27}$ K. C. Cheng, R. C. Lin, and J. W. Ou, "Fully developed laminar flow in curved rectangular channels,', J. Fluids Eng. 98, 41 (1976).

${ }^{28} \mathrm{~S}$. Thangam and N. Hur, "Laminar secondary flows in curved rectangular ducts,' J. Fluid Mech. 217, 421 (1990).

${ }^{29}$ K. N. Ghia and J. S. Sokhey, "Laminar incompressible viscous flow in curved ducts of regular cross-sections,', J. Fluids Eng. 99, 640 (1977).

${ }^{30}$ Y. Mori, Y. Uchida, and T. Ukon, "Forced convective heat transfer in a curved channel with a square cross section,' Int. J. Heat Mass Transfer 4, 1787 (1971)

${ }^{31}$ K. C. Cheng, R. C. Lin, and J. W. Ou, "Graetz problem in curved square channels,' J. Heat Transfer 97, 244 (1975).

${ }^{32}$ Y. Mori and Y. Uchida, "Study on forced convective heat transfer in a curved square channel (1st report, theory of laminar region),' Trans. J. Soc. Mech. Eng. 33, 1836 (1967).
${ }^{33} \mathrm{D} e$ is the usual Dean number based on the axial mean velocity $W_{m}$. Because of the change in friction resistance, it may vary even through the axial pressure gradient (also $D k$ ) is fixed. Its variation at a fixed $D k$ is an indication of the change in friction resistance.

${ }^{34}$ While the uniform grid spacing is used for all cases in the present work, the use of nonuniform mesh with appropriate distribution is desirable for the case of high rotation, strong curvature and heating/cooling in which a boundary layer appears near the wall and needs to be better resolved.

${ }^{35} \mathrm{~S}$. Yanase, N. Goto, and K. Yamamoto, "Stability of dual solutions of the flow in a curved circular tube,', J. Phys. Soc. Jpn. 57, 2602 (1988).

${ }^{36}$ E. M. Smirnov, "Asymptotic drag formulas for the rapidly rotating radial channels of rectangular cross section,' Izv. Akad. Nauk. SSSR. Mekh. Zhidk. Gaza, 42 (1978).

${ }^{37}$ C. G. Speziale, "Numerical study of viscous flow in rotating rectangular ducts,'’ J. Fluid Mech. 122, 251 (1982).

${ }^{38}$ W. H. Finlay, J. B. Keller, and J. H. Ferziger, "Instabilities and transition in curved channel flow,', J. Fluid Mech. 194, 417 (1988).

${ }^{39}$ W. H. Finlay, "Transition to oscillatory motion in rotating channel flow," J. Fluid Mech. 215, 209 (1990)

${ }^{40}$ P. M. Ligrani, W. H. Finlay, W. A. Fields, S. T. Fuqua, and C. S. Subramaman, "Features of wavy vortices in a curved channel from experimental and numerical studies,"' Phys. Fluids A 4, 695 (1992).

${ }^{41}$ P. Ligrani and R. D. Niver, "Flow visualization of Dean vortices in a curved channel with 40 to 1 aspect ratio," Phys. Fluids 31, 3605 (1988).

${ }^{42}$ Y. Guo and W. H. Finlay, "'Splitting, merging and wavelength selection of vortices in curved and/or rotating channel flow due to Eckhaus instability," J. Fluid Mech. 228, 661 (1991).

${ }^{43}$ P. A. Alfredsson and H. Persson, "Instabilities in channel flow with system rotation,', J. Fluid Mech. 202, 543 (1989).

${ }^{44}$ O. J. E. Matsson and P. H. Alfredsson, "Curvature- and rotation-induced instabilities in channel flow,', J. Fluid Mech. 210, 537 (1990).

${ }^{45}$ D. J. Tritton and P. A. Davies, "Instabilities in geophysical dynamics," in Hydrodynamic Instabilities and the Transition to Turbulence, 2nd ed., edited by H. L. Swinney and J. P. Gollub (Springer-Verlag, Berlin, 1985), Vol. 45, p. 229.

${ }^{46}$ L. Wang and K. C. Cheng, "Flow in curved channels with a low negative rotation speed,' Phys. Rev. E 51, 1155 (1995).

${ }^{47}$ L. Wang and K. C. Cheng, "Visualization of flows in curved channels with a moderate or high rotation speed,' Int. J. Rot. Mach. (in press).

${ }^{48}$ M. D. Hoover and W. Stöber, "Model of particle deposition in spinning duct aerosol centrifuges," J. Aerosol Sci. 12, 223 (1981).

${ }^{49}$ M. D. Hoover, W. Stöber, and G. Morawietz, "Experiment on laminar flow in a rotating, curved duct of rectangular cross section,' J. Fluid Eng. 106, 38 (1984).

${ }^{50}$ I. Motabazi, C. Normand, H. Peerhossaini, and J. E. Wesfreid, "Oscillatory modes in the flow between two horizontal corotating cylinders with a partially filled gap,', Phys. Rev. A 39, 763 (1989).

${ }^{51}$ O. J. E. Matsson, "Time-dependent instabilities in curved rotating channel flow,' Phys. Fluids A 5, 1514 (1993).

${ }^{52}$ B. Bara, K. Nandakumar, and J. H. Masliyah, "An experimental and numerical study of the Dean problem: Flow development towards twodimensional multiple solutions,', J. Fluid Mech. 244, 339 (1992).

${ }^{53}$ K. C. Cheng, J. Nakayama, and M. Akiyama, "Effect of finite and infinite aspect ratios on flow patterns in curved rectangular channels," in Flow Visualization, edited by T. Asanuma (Hemisphere, New York, 1979), pp. 181-186.

${ }^{54}$ S. Sugiyama, T. Hayashi, and K. Yamazaki, "Flow characteristics in the curved rectangular channels (visualization of secondary flow)," Bull. J. Soc. Mech. Eng. 26, 964 (1983). 